\title{
Effective inhibition of MYC-amplified Group 3 medulloblastoma through targeting translation machinery elF4F complex
}

\section{Yang Zhao}

Shanghai Jiaotong University School of Medicine Xinhua Hospital

Tiantian Li

Shanghai Jiao Tong University School of Medicine

\section{Shuaiwei Tian}

Shanghai Jiaotong University School of Medicine Xinhua Hospital

\section{Wei Meng}

Shanghai Jiaotong University School of Medicine Xinhua Hospital

\section{Jian Yang}

Shanghai Jiao Tong University School of Medicine

\section{Baocheng Wang}

Shanghai Jiaotong University School of Medicine Xinhua Hospital

\section{Zhuangzhuang Liang}

Shanghai Jiao Tong University School of Medicine

\section{Heng Zhao}

Shanghai Jiao Tong University School of Medicine

\section{Yipeng Han}

Shanghai Jiaotong University School of Medicine Xinhua Hospital

\section{Yujie Tang}

Shanghai Jiao Tong University School of Medicine

Jie Ma ( $\square$ majie@xinhuamed.com.cn)

Xin Hua Hospital affiliated to Shanghai Jiao Tong University School of Medicine

\section{Research}

Keywords: elF4F complex, elF4A1, MYC-amplified group 3 medulloblastoma, Silvestrol, translation inhibition

Posted Date: March 25th, 2020

DOI: https://doi.org/10.21203/rs.3.rs-19096/v1 
License: (c) (i) This work is licensed under a Creative Commons Attribution 4.0 International License. Read Full License 


\title{
Effective inhibition of MYC-amplified Group 3 medulloblastoma
} through targeting translation machinery eIF4F complex

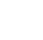

Yang Zhao ${ }^{{ }^{\dagger}}$, Tiantian $\mathrm{Li}^{2}$, Shuaiwei $\operatorname{Tian}^{{ }^{\dagger}}{ }^{\dagger}$, Wei Meng ${ }^{1}$, Jian Yang ${ }^{1}$, Baocheng Wang ${ }^{1}$, Zhuangzhuang Liang ${ }^{1}$, Heng Zhao ${ }^{1}$, Yipeng Han $^{1}$, Yujie Tang ${ }^{1,2^{*}}$, Jie Ma $^{1^{*}}$ (1)

1. Department of Pediatric Neurosurgery, Xin Hua Hospital Affiliated with Shanghai Jiao Tong University School of Medicine, Shanghai, 200092, People's Republic of China.

2. Key Laboratory of Cell Differentiation and Apoptosis of the National Ministry of Education, Department of Pathophysiology, Shanghai Jiao Tong University School of Medicine, 280 South Chongqing Road, Shanghai, 200025, People's Republic of China.

*Correspondence: yujietang@shsmu.edu.cn; majie@xinhuamed.com.cn ${ }^{\dagger}$ Yang Zhao, Tiantian Li, and Shuaiwei Tian contributed equally to this work.

\begin{abstract}
:
Background: Medulloblastoma (MB) consists of four major molecular subtypes and group 3 subtype (G3) is the most aggressive one. MYC amplification tends to happen in $\mathrm{G} 3 \mathrm{MB}$ and those patients exhibit the worse prognosis and need novel effective therapeutic approach most urgently. MYC has been shown to be the driver as well as crucial dependency for MYC-amplified G3-MB, thus serving as a promising therapeutic target.
\end{abstract}

Methods: In this study, we tested inhibiting MYC translation in MYC-amplified G3-MB by targeting eIF4F complex. We analyzed multiple MB tumor data sets to 
1 identify potential therapeutic target of eIF4F components and utilized genetic or

2 pharmacological approaches for validation. The relevant inhibitory mechanisms were

3 further investigated by Western blot and whole lysate mass spectrometry assays to

4 confirm the targeting of MYC translation.

5 Results: Tumor data set analyses identified eIF4A1, a subunit of eIF4F complex,

6 which was significantly upregulated in G3-MB compared to normal cerebellum and

7 highly correlated with MYC at transcript level. Targeting eIF4A1 with CRISPR/Cas9

8 approach or small-molecule inhibitor Silvestrol effectively attenuated growth of

9 multiple preclinical models through blocking proliferation and inducing apoptosis.

10 Mechanistically, Silvestrol treatment effectively inhibited MYC expression at protein

11 level and MYC amplification was shown to sensitize MB cells to eIF4A1 inhibition.

12 Moreover, whole proteome mass spectrometry analyses demonstrated additional

13 biological functions or pathways affected by eIF4A1 inhibition, which could help in

14 better understanding its inhibitory mechanisms and revealing combinatory strategies.

15 Conclusion: It is shown in our study that targeting MYC translation by eIF4A1

16 inhibition could be an effective therapeutic strategy against MYC-amplified G3-MB.

18 Keywords: eIF4F complex, eIF4A1, MYC-amplified group 3 medulloblastoma,

19 Silvestrol, translation inhibition

21 Running Title: Inhibition of MYC-amplified G3-MB by targeting eIF4F complex. 


\section{Background}

Medulloblastoma (MB) is among the most common malignant pediatric brain tumors, whose treatments currently include surgery, craniospinal radiation, high-dose cytotoxic chemotherapy, etc. In spite of all the aggressive therapies, still about one-third of patients die of their diseases, while the survivors suffer a lot from devastating side-effects, such as endocrine disorders, cognitive deficits, and higher morbidity of secondary cancers later in life[1]. In this sense, more effective and less toxic therapies are in desperate need for MB. Historically, the classification of MB was based on histopathology, with "classic" tumors having an average risk, desmoplastic/nodular tumors a more favorable prognosis, and large cell/anaplastic tumors very poor outcomes. Recent studies have shown that a genomics-based classification (i.e., gene expression, $\mathrm{CpG}$ methylation, DNA copy number, and mutations) may better predict prognosis and provide valuable information of potential drivers and therapies[2]. Despite its considerable genomic heterogeneity, there is a general consensus that $\mathrm{MB}$ can be divided into four major subgroups: Wingless (WNT), Sonic hedgehog (SHH), group 3, and group 4[3], with WNT having an extremely favorable prognosis, $\mathrm{SHH}$ and group 4 intermediate outcomes, and group 3 higher risk of relapse and death.

Approximately Group 3 tumor accounts for a quarter of MBs and has a peak incidence in childhood, twice as common in males as in females. It often demonstrates large-cell/anaplastic histopathology and has an elevated expression of c-MYC, with a high-level amplification of the MYC locus on chromosome $8 \mathrm{q}$ in $10 \%-20 \%$ of group 3 patients[1]. The amplified region often encodes a fusion between MYC and PVT1, a noncoding gene with a family of microRNAs that may promote transformation by cooperating with MYC[4]. Patients with MYC amplification are most susceptible to relapse and suffer from worst prognosis of all MB patients. Therefore, they are in most urgent need for novel effective therapies. MYC has been demonstrated to be an oncogenic driver as well as a crucial cancer-dependent gene of MYC-amplified G3-MB[5, 6], making it a promising therapeutic target. It has been a rather difficult task to pharmacologically target 
1 transcription factor MYC directly. However, an indirect targeting MYC at 2 transcription, translation or post-translation levels has recently shown promising 3 efficacy against multiple MYC-driven tumor types including MYC-amplified $4 \quad \mathrm{G} 3-\mathrm{MB}[7,8]$.

5 One common feature of neoplasia is the dysregulation of mRNA translation. Many translation initiation factors were found to be dysregulated in various cancers, including the subunits of the eukaryotic translation initiation factor 4F (eIF4F) complex[9-11], which regulates the cap-dependent mRNA translation process. The eIF4F complex comprises three components: eIF4E binds the 5' cap; the eIF4A RNA helicase unwinds the secondary structure; and eIF4G scaffolds other eIFs and enhances the eIF4A helicase activity. Overexpression of eIF4E promotes cell transformation[12] and frequently correlates with high tumor malignancy as well as unfavorable prognosis[13-15]. Elevated eIF4A and eIF4G levels have been reported in lung and hepatocellular carcinomas respectively[16-18], though the oncogenicity of eIF4A and eIF4G has not been as well characterized as eIF4E. Meanwhile, malignant progression in an acute lymphocytic leukemia mouse model can be enhanced by forced overexpression of eIF4A[19]. Furthermore, its enhanced protein translation has been verified in a spectrum of neurological disorders such as fragile $\mathrm{X}$ syndrome and autism spectrum disorders[20-22], suggesting the therapeutic potential for targeting translation initiation.

Translation initiation eIF4F complex is a heterotrimeric one which contains eIF4A, eIF4G, and eIF4E. It initiates mRNA translation with assembly of the complex at the m7G cap structure of the message, through which eIF4G forms the backbone of the complex, and combines cap-binding protein, eIF4E, with the RNA helicase, eIF4A[23]. The aberrant activity of the eIF4F complex, which regulates the cap-dependent mRNA translation process, is increasingly observed in many cancers leading to the selective synthesis of proteins involved in tumor growth and metastasis[24]. Direct inhibition of eIF4F is being studied in preclinical and clinical settings as well[24]. The eIF4A inhibitors have phenomenal anti-neoplastic effects particularly, whose pharmacodynamics for further use in the clinic is still in 
1 progress[23]. However, the role of translation inhibition hasn't been fully explored in G3 MB.

In this study, we confirmed that the protein stability of MYC was extremely low in MYC-amplified G3-MB cells, which is consistent with previous findings in other tumor types, so we aimed to find potential anti-eIF4F therapeutic approach of targeting MYC translation in MYC-amplified G3-MB.

\section{Materials and methods}

\section{Compounds and cell lines}

Silvestrol (HY-13251), Rocaglamide (HY-19356) were purchased from MedChemExpress (MCE). D425, D458, MB002 and HDMB03 cell lines were kindly provided by Prof. Yoon-Jae Cho (Oregon Health \& Science University). Human fetal neural stem cell line (hfNSC) was kindly provided by Prof. Michelle Monje (Stanford University School of Medicine). Daoy and 293T cell lines were obtained from Cell Bank of Chinese Academy of Science (Shanghai, China). D425, D458, D283, Daoy, UW228 and 293T were cultured in Dulbecco's modified Eagle's medium /High glucose (DMEM, BI-01-052-1ACS, Biological Industries) supplemented with 10\% fetal bovine serum (FBS, F2442, Sigma). MB002, HD-MB03 and hfNSC were cultured in Tumor Stem Media (TSM) comprising DMEM/F12 (11330-032, Gibco), B27(-A) (12587-010, Gibco), Neurobasal(-A)(10888-022, Gibco), human EGF (20 ng/ml, 100-15, PeproTech, NJ, USA), human FGF-basic (20 ng/ml, 100-18B, PeproTech), human LIF (10 ng/ml, 300-05, PeproTech), 1×Antibiotic-Antimycotic (15240-062, Gibco), and 0.2\% Heparin Solution (10 ng/ml, 07980, Stem Cell Technology).

Astrocytes were obtained from P1 neonatal mouse cortex. Tissue was dissociated into single cells by TypLE at $37^{\circ} \mathrm{C}$ for $15 \mathrm{~min}$. To remove the fibroblasts, cells were cultured in the uncoated dish for $30 \mathrm{~min}$ at $37^{\circ} \mathrm{C}$, and then astrocytes were transferred from the supernatant to a PDL-coated flask in DMEM supplemented with 10\% FBS and $1 \times$ penicillin-streptomycin solution. Granule neuron were obtained from the differentiated granule neuron precursors (GNPs). Cerebella from euthanized mice (P1) 
1 was dissociated into single cells by TrypLE for $15 \mathrm{~min}$ at $37^{\circ} \mathrm{C}$. Then, $35 \% / 65 \%$ gradient percoll solution was used to isolate GNPs. NB-B27 medium comprising $1 \times$ B27 (-A), $1 \mathrm{mM}$ sodium pyruvate, Neurobasal (-A) medium, 1\% FBS, 1×Antibiotic-Antimycotic, $1 \mathrm{mM}$ glucose (sigma), $1 \mathrm{mM} \mathrm{KCl}$ (sigma), and $2 \mathrm{mM}$ L-glutamine, was used to induce GNPs differentiate into Granule neuron in the $0.1 \%$ matrigel-coated 96-well plate (354230, BD) for 3-5 days.

\section{Cell proliferation, apoptosis and viability assays}

The Click-iT EdU Alexa Fluor 647 Flow Cytometry Assay Kit (C10640, Invitrogen, CA, USA) was used to evaluate the cell proliferation. Cells were treated with drug for $20 \mathrm{hr}$ and exposed to $10 \mu \mathrm{M}$ EdU for $8 \mathrm{hr}$. The positive degree of EdU shows the proliferating cell quantity.

Cell apoptosis was evaluated using the Annexin V-FITC Apoptosis Detection Kit I (556547, BD Biosciences, CA, USA). Cells were treated with drug for $48 \mathrm{hr}$ and exposed to $1 \mathrm{mg} / \mathrm{ml}$ DAPI (D9542, Sigma) and Annexin V. The FACS analyses were performed with the help of the BD Fortessa FACS machine (BD Biosciences, CA, USA). The data were measured with FlowJo software (FlowJo, LLC, OR, USA).

For cell viability assays, cells were placed in 96-well plates in triplicate (5000 cells/well). After drug treatment, cells were then subjected to Celltiter Glo assay (G7571, Promega, WI, USA) to measure the cell viability. Synergy H4 Hybrid Reader (BioTek, Winooski, VT, USA) was used to collect the data.

\section{Western Blots}

Cell lysates were obtained from samples with RIPA buffer containing $1 \mu \mathrm{M}$ protease inhibitor PMSF (BCBQ7707V, Sigma). Protein concentration was evaluated with Pierce $^{\mathrm{TM}}$ Bicinchoninic Acid (BCA) Protein Assay Kit (23225, Thermo Fisher Scientific) in accordance with the manufacturer's protocol. Equal amount protein was loaded for Western blot analysis with following primary antibodies: c-MYC (1: 1000, 5605S, Cell Signaling Technology), OTX2 (1:1000, 13497-1-AP, ProteinTech), eIF4A1 (1:1000, 2490s, Cell Signaling Technology), Beta-Tubulin (1: 5000, ab6046, 
Abcam).

\section{Plasmid construction, lentivirus preparation and infection}

The full length CDS of c-MYC was PCR-amplified from cDNA of D425 cells. ShRNAs against MYC oligonucleotides were annealed and cloned into pLKO.1-puro plasmid, while SgRNAs against eIF4A1 oligonucleotides into LentiCRISPR V2 plasmid in the same way. Lentivirus was generated by co-transfection of $293 \mathrm{~T}$ cells with transducing and packaging vectors psPAX and pMD2.G. Lentiviral particles were concentrated by PEG6000 and resuspended in PBS. The shRNA and sgRNA sequences were listed in supplementary.

\section{Patient-Derived Xenografts and Bioluminescence imaging of mice}

D425 cells were infected with sgeIF4A1 and sgScr lentivirus and implanted directly into the cerebellum of nude mice. In vivo experimental procedures were approved by the Experimental Animal Care Committee of Shanghai Jiao Tong University School of Medicine. Tumor-bearing mice were first subjected to bioluminescence imaging day 3 after transplantation and twice a week from that day on. For bioluminescence imaging (BLI), D-luciferin (YEASEN, Shanghai, China) was injected into anesthetized mice and in order to capture bioluminescence images (Xenogen IVIS, PerkinElmer, Waltham, MA, USA).

\section{Proteome analysis:}

A high-intensity ultrasonic processor (Scientz) in lysis buffer ( $8 \mathrm{M}$ urea, 1\% Protease Inhibitor Cocktail) was used to sonicate the sample three times on ice. (Note: For PTM experiments, inhibitors were also added to the lysis buffer, e.g. $3 \mu \mathrm{M}$ TSA and $50 \mathrm{mM}$ NAM for acetylation.) The remaining debris was disposed of by centrifugation at $12,000 \mathrm{~g}$ at $4{ }^{\circ} \mathrm{C}$ for $10 \mathrm{~min}$. Finally, the supernatant was collected and the protein concentration measured with BCA kit according to the manufacturer's instructions. 
1 at $56{ }^{\circ} \mathrm{C}$ and alkylated with $11 \mathrm{mM}$ iodoacetamide for $15 \mathrm{~min}$ at room temperature in darkness. Then $100 \mathrm{mM}$ TEAB was added to dilute the protein sample to urea concentration less than 2M. Finally, trypsin was added at 1:50 trypsin-to-protein mass ratio for the first digestion overnight and 1:100 trypsin-to-protein mass ratio for a second 4 h-digestion. The tryptic peptides were dissolved in $0.1 \%$ formic acid (solvent A) and directly loaded onto a home-made reversed-phase analytical column (15-cm length, $75 \mu \mathrm{m}$ i.d.). The gradient was comprised of an increase from $6 \%$ to $23 \%$ solvent B (0.1\% formic acid in $98 \%$ acetonitrile) over $26 \mathrm{~min}, 23 \%$ to $35 \%$ in $8 \mathrm{~min}$ and climbing to $80 \%$ in $3 \mathrm{~min}$ then holding at $80 \%$ for the last $3 \mathrm{~min}$, all at a constant flow rate of $400 \mathrm{~nL} / \mathrm{min}$ on an EASY-nLC 1000 UPLC system.

The peptides were subjected to NSI source followed by tandem mass spectrometry (MS/MS) in Q ExactiveTM Plus (Thermo) coupled online to the UPLC. The electrospray voltage applied was $2.0 \mathrm{kV}$. The m/z scan range was 350 to 1800 for full scan, and intact peptides were detected in the Orbitrap at a resolution of 70,000. Peptides were then selected for MS/MS using NCE setting as 28 and the fragments were detected in the Orbitrap at a resolution of 17,500. Automatic gain control (AGC) was set at 5E4. Fixed first mass was set as $100 \mathrm{~m} / \mathrm{z}$.

The resulting MS/MS data were processed with Maxquant search engine (v.1.5.2.8). Tandem mass spectra were searched against human uniprot database concatenated with reverse decoy database. Trypsin/P was specified as cleavage enzyme allowing up to 4 missing cleavages. The mass tolerance for precursor ions was set as $20 \mathrm{ppm}$ in First search and 5 ppm in Main search, while the mass tolerance for fragment ions was set as $0.02 \mathrm{Da}$. Carbamidomethyl on Cys was specified as fixed and acetylation modification and oxidation on Met were specified as variable ones. FDR was adjusted to $<1 \%$ and minimum score for modified peptides was set $>40$.

Gene Ontology (GO) annotation proteome was obtained from the UniProt-GOA database ( http://www.ebi.ac.uk/GOA/). If some identified proteins were not annotated by UniProt-GOA database, the InterProScan soft would be used to annotated protein's GO functional based on protein sequence alignment method. 


\section{Results}

Tumor data set analyses identifies eIF4A1 as a potential therapeutic target of MYC-amplified G3-MB.

Through a ubiquitin-proteasome pathway MYC can turn over rapidly. The E3 ubiquitin ligase SCF-FBXW7 binds and targets MYC for degradation after phosphorylation of MYC by GSK3 $\beta[25]$. To identify the low stability of MYC, we subjected MYC-amplified MB cells to Cycloheximide (CHX: a translation inhibitor) with augmentant time. A rapid decrease of MYC protein level can be observed after CHX treatment (as early as $2 \mathrm{~h}$ ), suggesting the low protein stability of MYC and the therapeutic potential for targeting MYC translation (Figure. 1A). Translation initiation is regulated by eukaryotic translation initiation factor 4F complex (eIF4F complex), comprising the eIF4E cap-binding protein, the eIF4A DEAD box RNA helicase, and the eIF4G scaffolding protein. The dependency score of eIF4A1 was the highest in 4 G3-MB cell lines (Figure 1 B). We investigated the correlation between MYC and the subunits of eIF4F in Cavalli database (Figure $1 \mathrm{E}$ ), and the differential expression of eIF4F subunits in subgroups of $\mathrm{MB}$ in pomeroy database (Figure 1C) or u133p2 database (Figure 1D). Analysis results revealed that only eIF4A correlated with MYC highly $(\mathrm{R}$ value $=0.614)$ meanwhile had elevated expression in MYC-amplified MB specifically (Fig. 1C,D). Based on these results, we made assumption that direct targeting eIF4A may be a prospective strategy to treat MYC-amplified MB.

\section{Genetically targeting eIF4A1 inhibits growth of MYC-amplified G3-MB in vitro} and in vivo.

In line with this assumption, we tested whether knockdown of eIF4A could decrease tumor cell proliferation and viability in vitro and in vivo. We targeted eIF4A by CRISPR-Cas9 approach could against D425, D458 and MB002 cells. Our results showed that knockout of eIF4A in MYC-amplified MB lines resulted in significantly disruption of tumor cell growth in vitro (Fig. 2A B). Accordantly, nude mice orthotopically xenografted with eIF4A1-knockout D425 cells showed dramatically improved survival $(p=0.0324)$ and tumer size compared to the ones with sgGFP D425 
1 cells (Fig. 2C-E). These data indicated that eIF4A was integrant for tumor growth of

2 MYC-amplified MB both in vitro and in vivo, thus confirming its potential as

3 therapeutic drug target.

4

eIF4A inhibitor silvestrol attenuates growth of MYC-amplified G3-MB in vitro through blocking proliferation and inducing apoptosis

To further demonstrate the effect of targeting eIF4A in MYC-amplified MB, we therefore tried an alternative to inhibit eIF4A by adopting the compound silvestrol, which increases the affinity between eIF4A and RNA, thereby sequestering and depleting eIF4A from translation initiation complexes [24, 26]. We exposed G3 MB cell lines and control cell lines to eIF4A1 inhibitors silvestrol and ROC-A and found that G3 MB cell lines much more sensitive to these inhibitors (Fig. 3A). Timecourse study showed that silvestrol inhibit the growth of G3 MB cells in a time-and-dose-dependent manner (Fig. 3B). Our flowcytometry results showed that silvestrol induced significant cell apoptosis and greatly suppressed cell proliferation in G3 MB cells (Fig. 3 C,D). These results suggest that eIF4A1 inhibitor could significantly suppresses G3 MB cell growth.

Silvestrol effectively inhibits MYC translation and MYC amplification is a determinant of its sensitivity

With a view to exploring the inhibition of eIF4A1i on MYC expression, the q-PCR results showed that mRNA levels of MYC in D425, D458 and MB002 cells treated with ROC-A or silvestrol were elevated (Fig. 4D) suggesting eIF4Ai didn't take effect in mRNA levels. Then immunoblot was applied to detect MYC, OTX2 expression of D425, MB002 and HDMB03 cells treated with Silvestrol (10 ng/mL) and results showed that eIF4A1i downregulate the MYC protein level over time (Fig. 4A-C). So next we want to see whether the level of MYC contribute to the functional effects of Silvestrol. Cell viability was detected in MB002 cells transfected with shMYC or scrambled shRNA (shScr) (Fig. 4E), or in Daoy cells overexpressing MYC or empty 
1 vector (EV) (Fig. 4F).The exogenous expression of MYC increased the effect of

2 Silvestrol, and on the opposite, knockdown MYC decreased its effect on cell viability,

3 suggesting that level of MYC contribute to the functional effects of Silvestrol.

4

Whole proteome analyses identified additional biological functions or pathways affected by eIF4A1 inhibition in MYC-amplified G3-MB cells.

In order to explore the pattern of preteomic modulation of silvestrol treatment in $\mathrm{MB}$ cells, HDM03 cells treated with DMSO or Sil were send for QC-WB or proteomic analysis at the same time (Fig. 5A). The proteomic analysis results indicated that the proteomic expression of MB was significantly modulated by Silvestrol with most of genes down-regulated (Fig. 5C). The dependency scores of these genes were mostly below zero suggesting these genes were mostly important for MB cell growth (Fig. 5C). The function of proteins down-regulated by silvestrol in MYC-amplified G3-MB cells were mostly related to cell cycle regulation and heat shock response (Fig. 5D), and the function of proteins up-regulated by silvestrol were mostly related to mitochondrial translation and fatty acid metabolism. (Fig. 5E)

\section{Discussion}

Targeting translation machinery has been shown as an effective therapeutic strategy against multiple tumor types. Direct inhibition of eIF4F complex is being investigated in preclinical and clinical settings (i.e., eIF4E antisense oligonucleotides[27-29], inhibitors of eIF4E-5'-mRNA-cap and eIF4E-eIF4G interaction[30], and inhibitors of eIF4A[31, 32]). In particular, eIF4A inhibitors display impressive anti-neoplastic effects, with efforts underway to improve their pharmacodynamics for use in the clinic. In this study, we try to overcome MYC-amplified MB by targeting the translational machine of MYC. Tumor data set analyses identified eIF4A1, a subunit of eIF4F complex, was significantly upregulated in G3-MB compared to normal cerebellum and highly correlated with MYC at transcript level. Targeting eIF4A1 with CRISPR/Cas9 approach or small-molecule inhibitor Silvestrol effectively attenuated 
1 growth of multiple preclinical models through blocking proliferation and inducing apoptosis. Mechanistically, Silvestrol treatment effectively inhibited MYC expression at protein level and MYC amplification was shown to sensitize MB cells to eIF4A1 inhibition. Moreover, whole proteome mass spectrometry analyses demonstrated additional biological functions or pathways affected by eIF4A1 inhibition, which could help better understanding its inhibitory mechanisms and revealing combinatory strategies.

There are three members in the MYC family, including MYC, MYCN, and MYCL1, whose amplifications can be found in the subtypes of medulloblastoma[33]. Group 3 tumors are often associated with amplification of MYC. Group 3 tumors without MYC amplification are often characterized by overexpression of MYC or amplification of MYCN[34]. MYCL1 amplifications have been found in a few SHH tumor cases whereas $\mathrm{SHH}$ and group 4 tumors are liable to be associated with amplifications of MYCN. TP53 mutation often occurs with MYCN amplification in SHH subtype, whose patients are also with the worst prognosis[33, 34]. Since MYCN has similar properties to MYC, eIF4A inhibition may also have therapeutic effect on this type of tumor. However, we are not able to test it for the time being due to the lack of appropriate human PDC and PDX models.

Whole proteome analyses of silvestrol-treated MYC-amplified G3-MB cells demonstrated that anti-eIF4A induced translation inhibition could significantly downregulate heat shock response related functions, which is consistent with a previous study saying that eIF4A inhibition inhibits HSF1 transactivation activity in cancer cells $[35,36]$. Importantly, that study also shows that hyperactive translation in cancer cells could induce HSF1 transactivation to reprogram cancer transcriptome to support protein production in a positive feed-back way, including the modulation of cancer metabolism[36]. Therefore, we plan to further investigate this regulatory axis in MYC-amplified G3-MB and identify other potential therapeutic targets in it. Whole proteome analyses also found that mitochondrial translation and fatty acid metabolism were the top upregulated functions by eIF4A inhibition. Intriguingly, these two functions have been shown to contribute to drug resistance before in many other 
1 cancer types[37-40], thus our results suggest that they may also affect the efficacy of

2 eIF4A inhibition in MYC-amplified G3-MB and it would be interesting to test the

3 combination of targeting these two functions with eIF4A inhibition.

4

\section{Conclusion}

6 Our study demonstrates that targeting MYC translation by eIF4A1 inhibition could be

7 an effective therapeutic strategy against MYC-amplified G3-MB. As the flavagline

8 class anti-eIF4A1 small-molecule inhibitors (such as Silvestrol and Roc-A) that are

9 currently widely used in research have not be convincingly proven to efficiently cross

10 blood-brain-barrier (BBB), still further efforts will be needed to translate our finding

11 from bench-side to bedside. 


\section{Abbreviations}

MB: Medulloblastoma; G3-MB: Group 3 medulloblastoma; SHH: Sonic Hedgehog; G3: Group3; eIF: eukaryotic translation initiation factor; DMEM: Dulbecco's modified Eagle's medium; FBS: feta bovine serum; GFP: Green fluorescence protein; FACS: Fluorescence-activated cell sorting; GO: Gene oncology. DAPI: 4',6-diamidino-2-phenylindole; DMSO: Dimethyl sulfoxide; FDR: False discovery rate.

\section{Ethics approval and consent to participate}

Not applicable.

\section{Consent for publication}

Not applicable.

\section{Availability of data and materials}

The datasets used and analyzed during the current study are available from the corresponding author upon request.

\section{Competing interests}

The authors declare that they have no competing interests.

\section{Funding}

This work was supported by the National Natural Science Foundation of China (81702453 to Yang Zhao, 81702939 to Yipen Han, 81572761 to Yujie Tang, 81772655 and 81972646 to Yujie Tang), Shanghai Science and Technology Committee Funding (No. 17411965700 to Yang Zhao, 17411951800 and 19411952100 to Jie Ma), Shanghai "Rising Stars of Medical Talent" Youth Development Program( [2019] No.72 to Yang Zhao), Shanghai Jiao Tong University Yi Gong Jiao Cha Funding (YG2015QN42 to Yang Zhao, YG2019QNA45 to Wei Meng), Shanghai Shen Kang Hospital Development Center (16CR2031B to Jie Ma), Chinese Universities Scientific Fund, the Recruitment Program of Global Experts of China (Yujie Tang), the Program for Professor of Special Appointment (Eastern Scholar) at Shanghai Institutions of Higher Learning (Yujie Tang), Shanghai Rising-Star Program (Yujie Tang).

\section{Authors' contributions}

Jie Ma and Yujie Tang designed the project and experiments. Tiantian Li, Yang Zhao, Shuaiwei Tian, Wei Meng and Jian Yang conducted the experiments. Yang Zhao, Tiantian Li, Yipeng Han, Zhuangzhuang Liang and Baocheng Wang analyzed the RNA-seq data, Yang Zhao, Wei Meng and Heng Zhao generated the figures and wrote the manuscript, and Jie Ma and Yujie Tang supervised all aspects of the work. All authors read and approved the final manuscript.

\section{Acknowledgements}


1 We thank all members of the Ma and Tang laboratories for their helpful discussions 2 and advice regarding this work.

3

4 Author details

$5 \quad{ }^{1}$ Department of Pediatric Neurosurgery, Xin Hua Hospital Affiliated with Shanghai 6 Jiao Tong University School of Medicine, Shanghai 200092, People's Republic of 7 China. ${ }^{2}$ Key Laboratory of Cell Differentiation and Apoptosis of the National 8 Ministry of Education, Department of Pathophysiology, Shanghai Jiao Tong 9 University School of Medicine, 280 South Chongqing Road, Shanghai 200025, 10 People's Republic of China. 


\section{References:}

1. Coluccia D, Figuereido C, Isik S, Smith C, Rutka JT: Medulloblastoma: Tumor Biology and Relevance to Treatment and Prognosis Paradigm. Curr Neurol Neurosci Rep 2016, 16:43.

2. Taylor MD, Northcott PA, Korshunov A, Remke M, Cho YJ, Clifford SC, Eberhart CG, Parsons DW, Rutkowski S, Gajjar A, et al: Molecular subgroups of medulloblastoma: the current consensus. Acta Neuropatho/ 2012, 123:465-472.

3. Northcott PA, Korshunov A, Witt H, Hielscher T, Eberhart CG, Mack S, Bouffet E, Clifford SC, Hawkins CE, French P, et al: Medulloblastoma comprises four distinct molecular variants. J Clin Oncol 2011, 29:1408-1414.

4. Tao R, Murad N, Xu Z, Zhang P, Okonechnikov K, Kool M, Rivero-Hinojosa S, Lazarski C, Zheng P, Liu Y, et al: MYC Drives Group 3 Medulloblastoma through Transformation of Sox2(+) Astrocyte Progenitor Cells. Cancer Res 2019, 79:1967-1980.

5. Pei Y, Moore CE, Wang J, Tewari AK, Eroshkin A, Cho YJ, Witt H, Korshunov A, Read TA, Sun JL, et al: An animal model of MYC-driven medulloblastoma. Cancer Cel/ 2012, 21:155-167.

6. Kawauchi D, Robinson G, Uziel T, Gibson P, Rehg J, Gao C, Finkelstein D, Qu C, Pounds S, Ellison DW, et al: $\mathbf{A}$ mouse model of the most aggressive subgroup of human medulloblastoma. Cancer Cel/ 2012, 21:168-180.

7. Mertz JA, Conery AR, Bryant BM, Sandy P, Balasubramanian S, Mele DA, Bergeron L, Sims RJ, 3rd: Targeting MYC dependence in cancer by inhibiting BET bromodomains. Proc Natl Acad Sci U S A 2011, 108:16669-16674.

8. Bolin S, Borgenvik A, Persson CU, Sundstrom A, Qi J, Bradner JE, Weiss WA, Cho YJ, Weishaupt $H$, Swartling FJ: Combined BET bromodomain and CDK2 inhibition in MYC-driven medulloblastoma. Oncogene 2018, 37:2850-2862.

9. Wang S, Darini C, Desaubry L, Koromilas AE: STAT1 Promotes KRAS Colon Tumor Growth and Susceptibility to Pharmacological Inhibition of Translation Initiation Factor elF4A. Mol Cancer Ther 2016, 15:3055-3063.

10. Rivero-Hinojosa S, Lau LS, Stampar M, Staal J, Zhang H, Gordish-Dressman H, Northcott PA, Pfister SM, Taylor MD, Brown KJ, Rood BR: Proteomic analysis of Medulloblastoma reveals functional biology with translational potential. Acta Neuropathol Commun 2018, 6:48.

11. Oblinger JL, Burns SS, Huang J, Pan L, Ren Y, Shen R, Kinghorn AD, Welling DB, Chang LS: Overexpression of elF4F components in meningiomas and suppression of meningioma cell growth by inhibiting translation initiation. Exp Neurol 2018, 299:299-307.

12. Lazaris-Karatzas A, Montine KS, Sonenberg $\mathrm{N}$ : Malignant transformation by a eukaryotic initiation factor subunit that binds to mRNA 5' cap. Nature 1990, 345:544-547.

13. Li BD, Liu L, Dawson M, De Benedetti A: Overexpression of eukaryotic initiation factor 4E (elF4E) in breast carcinoma. Cancer 1997, 79:2385-2390.

14. Berkel HJ, Turbat-Herrera EA, Shi R, de Benedetti A: Expression of the translation initiation factor elF4E in the polyp-cancer sequence in the colon. Cancer Epidemiol Biomarkers Prev 2001, 10:663-666. 
15. Li Y, Fan S, Koo J, Yue P, Chen ZG, Owonikoko TK, Ramalingam SS, Khuri FR, Sun SY: Elevated expression of eukaryotic translation initiation factor $4 \mathrm{E}$ is associated with proliferation, invasion and acquired resistance to erlotinib in lung cancer. Cancer Biol Ther 2012, 13:272-280.

16. Shuda M, Kondoh N, Tanaka K, Ryo A, Wakatsuki T, Hada A, Goseki N, Igari T, Hatsuse K, Aihara $T$, et al: Enhanced expression of translation factor mRNAs in hepatocellular carcinoma. Anticancer Res 2000, 20:2489-2494.

17. Bauer C, Diesinger I, Brass N, Steinhart H, Iro H, Meese EU: Translation initiation factor elF-4G is immunogenic, overexpressed, and amplified in patients with squamous cell lung carcinoma. Cancer 2001, 92:822-829.

18. Comtesse N, Keller A, Diesinger I, Bauer C, Kayser K, Huwer H, Lenhof HP, Meese E: Frequent overexpression of the genes FXR1, CLAPM1 and EIF4G located on amplicon 3q26-27 in squamous cell carcinoma of the lung. Int J Cancer 2007, 120:2538-2544.

19. Wolfe AL, Singh K, Zhong Y, Drewe P, Rajasekhar VK, Sanghvi VR, Mavrakis KJ, Jiang M, Roderick JE, Van der Meulen J, et al: RNA G-quadruplexes cause elF4A-dependent oncogene translation in cancer. Nature 2014, 513:65-70.

20. Robichaud N, Sonenberg N, Ruggero D, Schneider RJ: Translational Control in Cancer. Cold Spring Harb Perspect Bio/ 2019, 11.

21. Gkogkas CG, Khoutorsky A, Ran I, Rampakakis E, Nevarko T, Weatherill DB, Vasuta C, Yee S, Truitt M, Dallaire P, et al: Autism-related deficits via dysregulated elF4E-dependent translational control. Nature 2013, 493:371-377.

22. Gkogkas CG, Khoutorsky A, Cao R, Jafarnejad SM, Prager-Khoutorsky M, Giannakas N, Kaminari A, Fragkouli A, Nader K, Price TJ, et al: Pharmacogenetic inhibition of elF4E-dependent Mmp9 mRNA translation reverses fragile $X$ syndrome-like phenotypes. Cell Rep 2014, 9:1742-1755.

23. Malka-Mahieu H, Newman M, Desaubry L, Robert C, Vagner S: Molecular Pathways: The elF4F Translation Initiation Complex-New Opportunities for Cancer Treatment. Clin Cancer Res 2017, 23:21-25.

24. Bhat M, Robichaud N, Hulea L, Sonenberg N, Pelletier J, Topisirovic I: Targeting the translation machinery in cancer. Nat Rev Drug Discov 2015, 14:261-278.

25. Castell A, Larsson LG: Targeting MYC Translation in Colorectal Cancer. Cancer discovery 2015, 5:701-703.

26. Bordeleau ME, Robert F, Gerard B, Lindqvist L, Chen SM, Wendel HG, Brem B, Greger H, Lowe SW, Porco JA, Jr., Pelletier J: Therapeutic suppression of translation initiation modulates chemosensitivity in a mouse lymphoma model. The Journal of clinical investigation 2008, 118:2651-2660.

27. Rinker-Schaeffer CW, Graff JR, De Benedetti A, Zimmer SG, Rhoads RE: Decreasing the level of translation initiation factor $4 \mathrm{E}$ with antisense RNA causes reversal of ras-mediated transformation and tumorigenesis of cloned rat embryo fibroblasts. Int J Cancer 1993, 55:841-847.

28. De Benedetti A, Joshi-Barve S, Rinker-Schaeffer C, Rhoads RE: Expression of antisense RNA against initiation factor elF-4E mRNA in HeLa cells results in lengthened cell division times, diminished translation rates, and reduced levels of both eIF-4E and 
the p220 component of elF-4F. Mol Cell Biol 1991, 11:5435-5445.

29. DeFatta RJ, Nathan CO, De Benedetti A: Antisense RNA to elF4E suppresses oncogenic properties of a head and neck squamous cell carcinoma cell line. Laryngoscope 2000, 110:928-933.

30. Zochowska M, Piguet AC, Jemielity J, Kowalska J, Szolajska E, Dufour JF, Chroboczek J: Virus-like particle-mediated intracellular delivery of mRNA cap analog with in vivo activity against hepatocellular carcinoma. Nanomedicine 2015, 11:67-76.

31. Malina A, Mills JR, Pelletier J: Emerging therapeutics targeting mRNA translation. Cold Spring Harb Perspect Biol 2012, 4:a012377.

32. Bordeleau ME, Mori A, Oberer M, Lindqvist L, Chard LS, Higa T, Belsham GJ, Wagner G, Tanaka J, Pelletier J: Functional characterization of IRESes by an inhibitor of the RNA helicase eIF4A. Nat Chem Biol 2006, 2:213-220.

33. Northcott PA, Shih DJ, Peacock J, Garzia L, Morrissy AS, Zichner T, Stutz AM, Korshunov A, Reimand J, Schumacher SE, et al: Subgroup-specific structural variation across 1,000 medulloblastoma genomes. Nature 2012, 488:49-56.

34. Bandopadhayay P, Bergthold G, Nguyen B, Schubert S, Gholamin S, Tang Y, Bolin S, Schumacher SE, Zeid R, Masoud S, et al: BET bromodomain inhibition of MYC-amplified medulloblastoma. Clin Cancer Res 2014, 20:912-925.

35. de Billy E, Clarke PA, Workman P: HSF1 in Translation. Cancer Cel/2013, 24:147-149.

36. Santagata S, Mendillo ML, Tang YC, Subramanian A, Perley CC, Roche SP, Wong B, Narayan $\mathrm{R}$, Kwon $\mathrm{H}$, Koeva $\mathrm{M}$, et al: Tight coordination of protein translation and HSF1 activation supports the anabolic malignant state. Science 2013, 341:1238303.

37. Sharon D, Cathelin S, Mirali S, Di Trani JM, Yanofsky DJ, Keon KA, Rubinstein JL, Schimmer AD, Ketela T, Chan SM: Inhibition of mitochondrial translation overcomes venetoclax resistance in AML through activation of the integrated stress response. Sci Trans/ Med 2019, 11.

38. Dong Z, Abbas MN, Kausar S, Yang J, Li L, Tan L, Cui H: Biological Functions and Molecular Mechanisms of Antibiotic Tigecycline in the Treatment of Cancers. Int $J$ Mol Sci 2019, 20.

39. Iwamoto H, Abe M, Yang Y, Cui D, Seki T, Nakamura M, Hosaka K, Lim S, Wu J, He X, et al: Cancer Lipid Metabolism Confers Antiangiogenic Drug Resistance. Cel/ Metab 2018, 28:104-117 e105.

40. Cao Y: Adipocyte and lipid metabolism in cancer drug resistance. J Clin Invest 2019, 129:3006-3017. 


\section{Figure Legends}

\section{Figure 1. MYC is highly correlated with eIF4A1 in Group 3 MB.}

(A) Western blots for the protein levels of MYC and OTX2 in Group 3 MB cell lines HDMB03, MB002 and D425 treated with $50 \mathrm{ng} / \mathrm{mL}$ cycloheximide (CHX). Immunoblot of $\beta$-tubulin is used as a loading control. (B) The dependency score of genes in 4 G3-MB cell lines. (C) The box plots showing the $\log 2$ fold changes of mRNA expression levels of eIF4F complex members (from left to right: eIF4A1, eIF4E, eIF4G1, eIF4G2) compared normal cerebellum with different subtype MBs of Pomeroy database. (D) The box plots showing the $\log 2$ fold changes of mRNA expression levels of eIF4F complex members (from left to right: eIF4A1, eIF4E, eIF4G1, eIF4G2) compared normal cerebellum with different subtype MBs of U133p2 database. (E) Scatterplots for the expression correlation of MYC with different subunits of eIF4F complex of MB patients in Cavalli database $(n=763)$. Spearman rank correlation test, Rs $=$ Spearman rank correlation coefficient. All box plots are plotted as mean \pm S.D. ${ }^{*}, \mathrm{p}<0.05 ; * *, \mathrm{p}<0.01 ; * * *, \mathrm{p}<0.001$ by two-tailed Student's t test. MB, medulloblastoma.

Figure 2. Knock-out $e I F 4 A 1$ can inhibit $\mathrm{MB}$ cell proliferation in vitro and xenograft growth in vivo.

(A-B) CRISPR/Cas9-mediated deletion of the eIF4A1 inhibits D425 cell growth in vitro. (C-D) eIF4A1 knock-out (sgeIF4A1) or control (sgScr) D425 cells were inoculated orthotopically into the cerebellum of nude mice. BLI images of photon fluxes of brain tumors at different times were shown (C). The quantification of fluorescence fluxes in B was compared (D). The survival curve of mice bearing sgeIF4A1 or sgScr D425 MB tumors were shown (E). Mice implanted with sgeIF4A1 D425 tumors display longer survival time with no effect on weight. ( $\mathrm{n}=6$ for sgeIF4A1 group and $\mathrm{n}=5$ for $\mathrm{sgScr}$ group). Gehan-Breslow-Wilcoxon test for survival comparison. Two-tailed Student's t test for bioluminescence comparison. sgScr, scrambled sgRNA. 
1 Figure 3. Validation of the inhibitory effects of eIF4A1 inhibitor Silvestrol in

2 MYC-amplified MB. (A) Cell viability was detected in different type of cells after 3 treated with increasing doses of eIF4A1 inhibitor (left, Silvestrol; right, Roc-A). (B)

4 Cell viability of D425 (left) or HDMB03 (right) was detected when treated with

5 increasing doses of eIF4A1 inhibitor silvestrol for indicated times. (C-D) Apoptosis

6 and proliferation were measured by FACS flow cytometry. Pecentage of Annexin V 7 positive (C) or EdU positive (D) cells of Silvestrol treated $(0.005$ or $0.01 \mu \mathrm{M})$ 8 HDMB03 and D425 cells were shown. All experiments were repeated at least three 9 times with triplicate samples. Data are plotted as means \pm s.d. *, p $<0.05$; **, p $<0.01$; $10 * * *, \mathrm{p}<0.001$ by two-tailed Student's $t$ test.

Figure 4. MYC play an essential role in inhibition of MB treated with Silvestrol. (A) Immunoblot was applied to detect MYC, OTX2 expression of D425, MB002 and HDMB03 cells treated with Silvestrol $(10 \mu \mathrm{g} / \mathrm{mL})$ or CHX $(20 \mu \mathrm{g} / \mathrm{mL})$ for $0-4 \mathrm{hrs}$. (B) Immunoblot was applied to detect MYC, OTX2 expression of D425, MB002 and HDMB03 cells treated with Silvestrol $(10 \mu \mathrm{g} / \mathrm{mL})$ or CHX $(20 \mu \mathrm{g} / \mathrm{mL})$ for $0-24 \mathrm{hrs}$. (C) Immunoblot was applied to detect MYC, OTX2 expression of D425, MB002 and HDMB03 cells treated with Silvestrol $(10 \mu \mathrm{g} / \mathrm{mL})$ or ROC-A $(50 \mu \mathrm{g} / \mathrm{mL})$. (D) Q-PCR for mRNA levels of MYC in D425, D458 and MB002 cells treated with ROC-A or silvestrol. (E-F) Cell viability was detected in MB002 cells transfected with shMYC or scrambled shRNA (shScr) (D), or in Daoy cells overexpressing MYC or empty vector (EV) (E). Western blots for the protein levels of eIF4A1 in MB002 or Daoy cells expressing shRNAs against MYC (shMYC) or scrambled shRNA (shScr) were present at the left respectively. Beta-Tubulin was used as loading control.

Figure 5. The proteomic expression of MB was modulated by treatment with Silvestrol. (A) HDM03 cells treated with DMSO or Sil were send for QC-WB or 
1 proteomic analysis at the same time. (B) Western blot result of QC. (C) Heatmap of 2 proteomic analysis was shown left and the heatmap of gene dependency were shown 3 right. (D) The function analysis of genes down regulated by silvestrol. (E) The 4 function analysis of genes up regulated by silvestrol.

5

6

7 
Figure 1
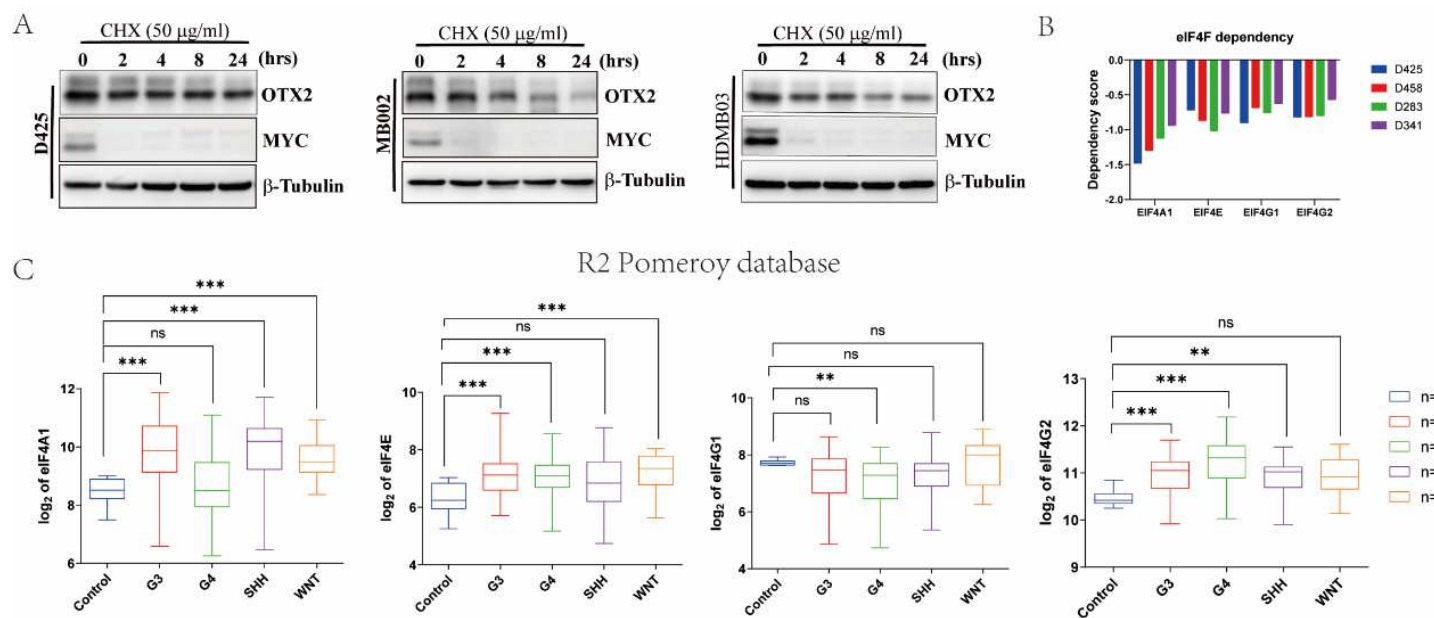

R2 Pomeroy database

D
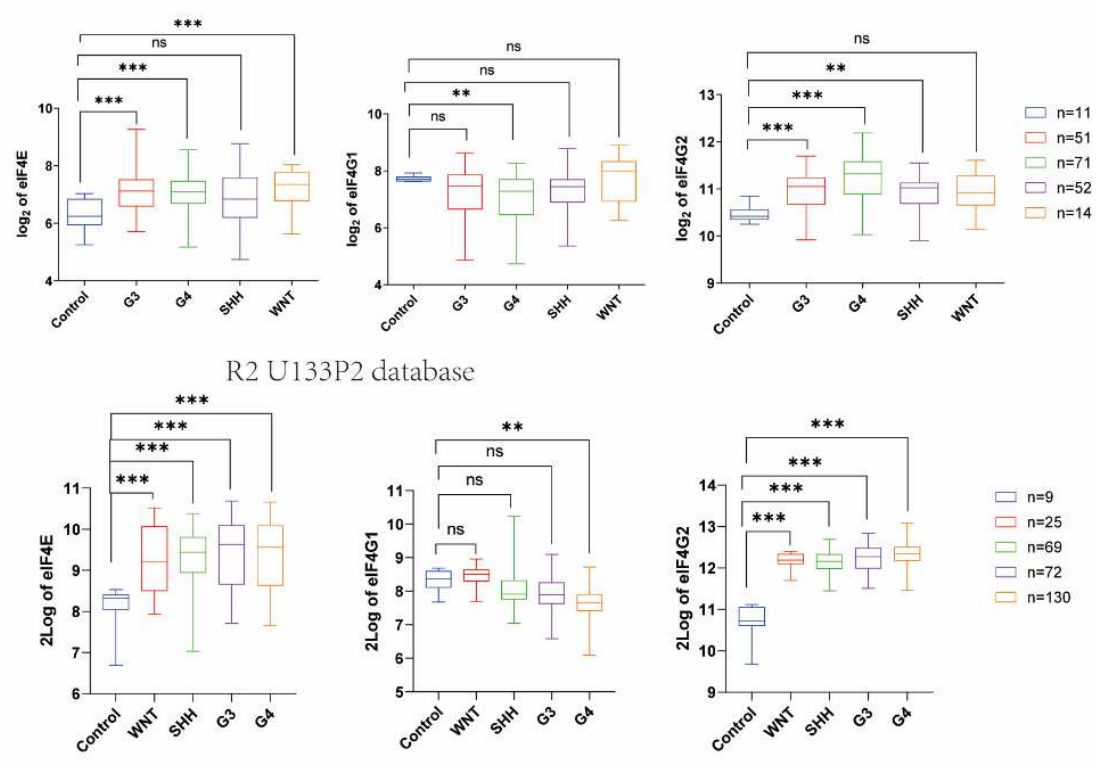

E
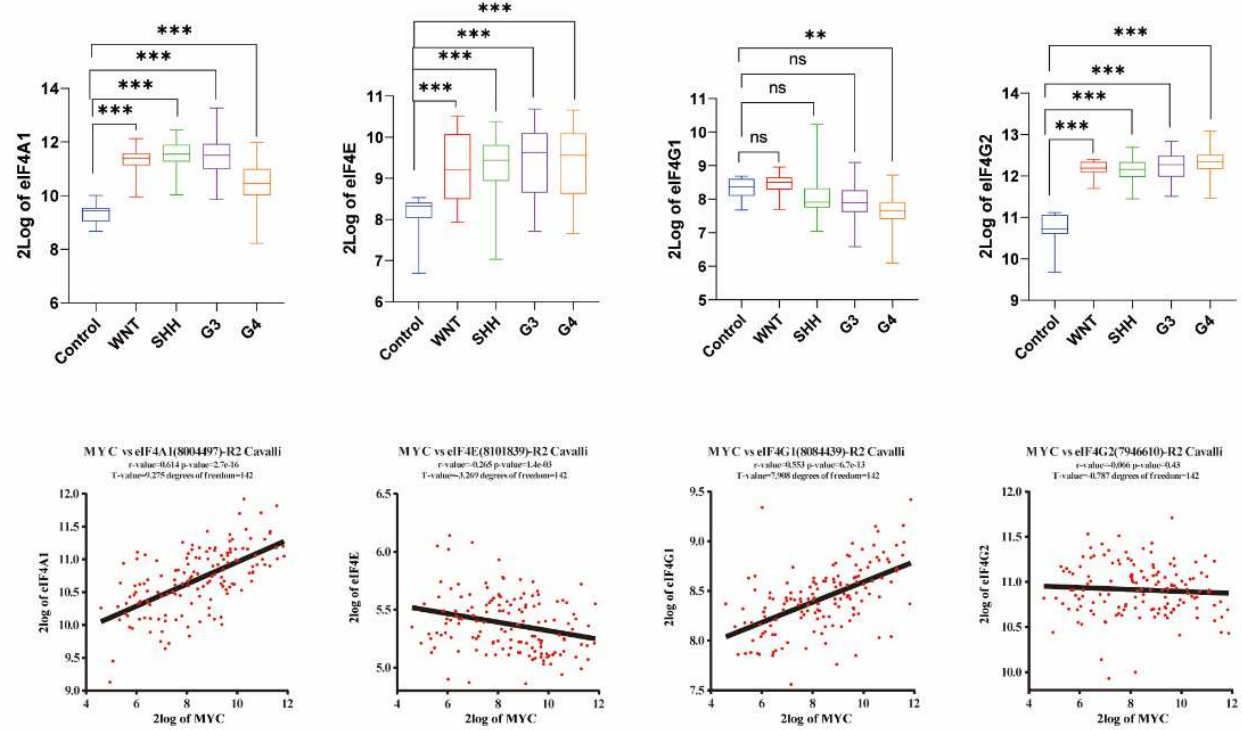
Figure 2
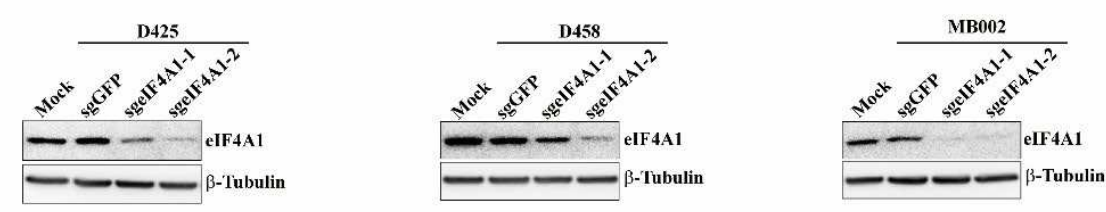

B
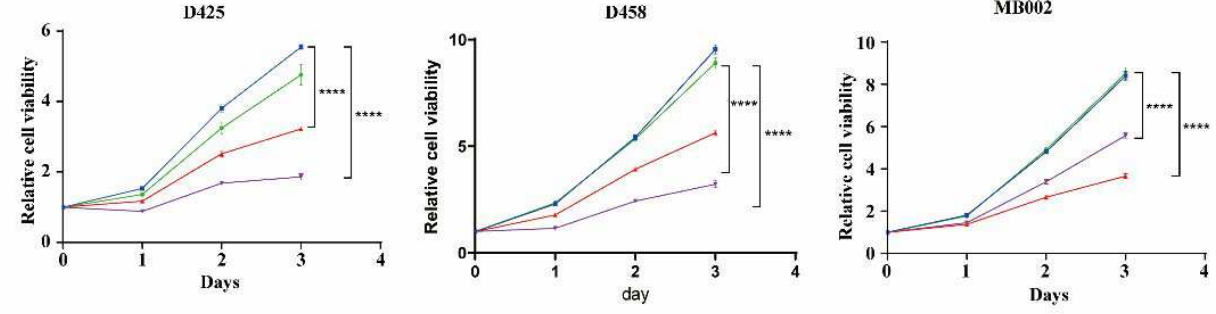

$\rightarrow$ Mock $\rightarrow$ sgSer $\rightarrow$ sgelF4A1-1 $\rightarrow$ sgelF4A1-2

C
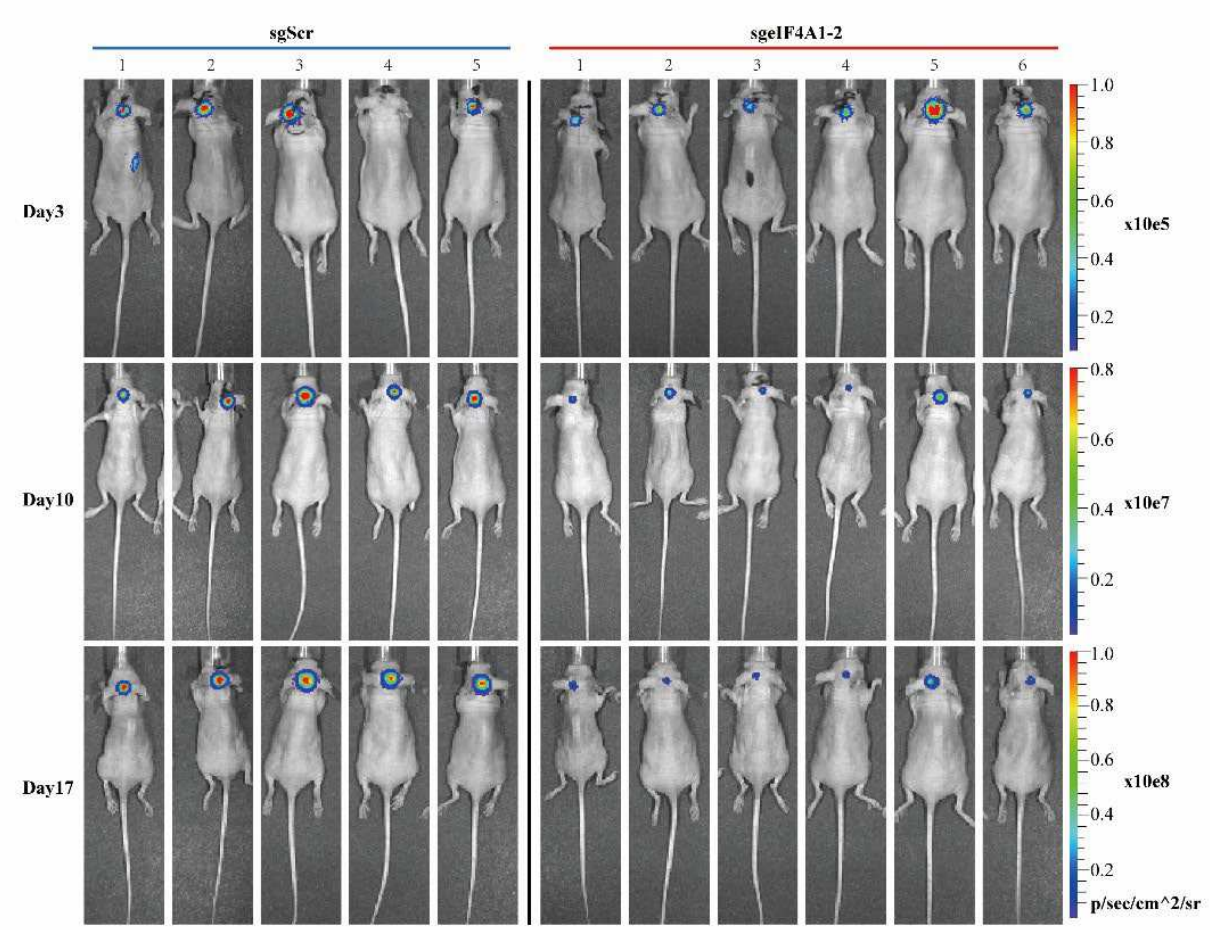

D

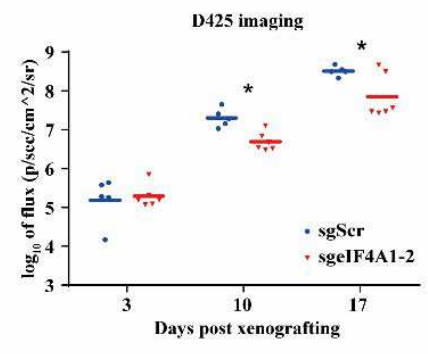

E

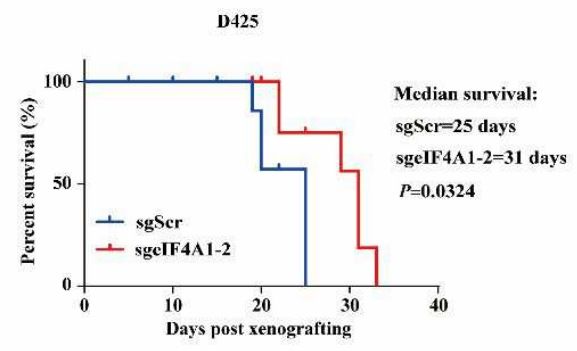


$1 \quad$ Figure 3

A

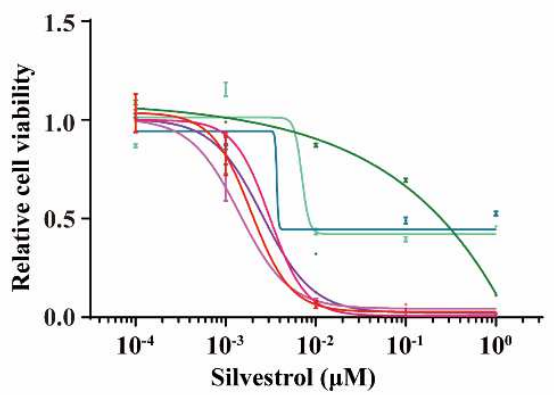

B

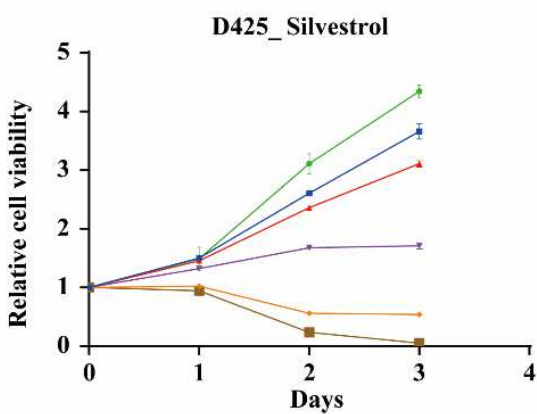

Roc-A
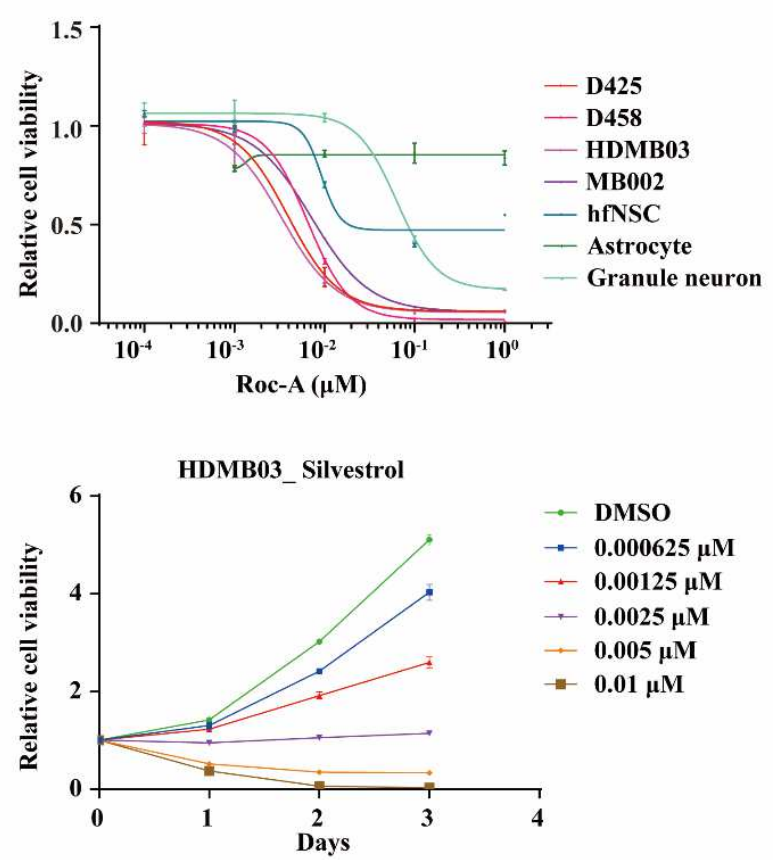

C

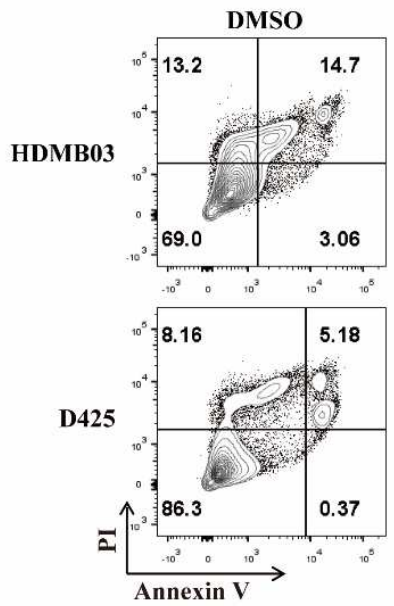

D
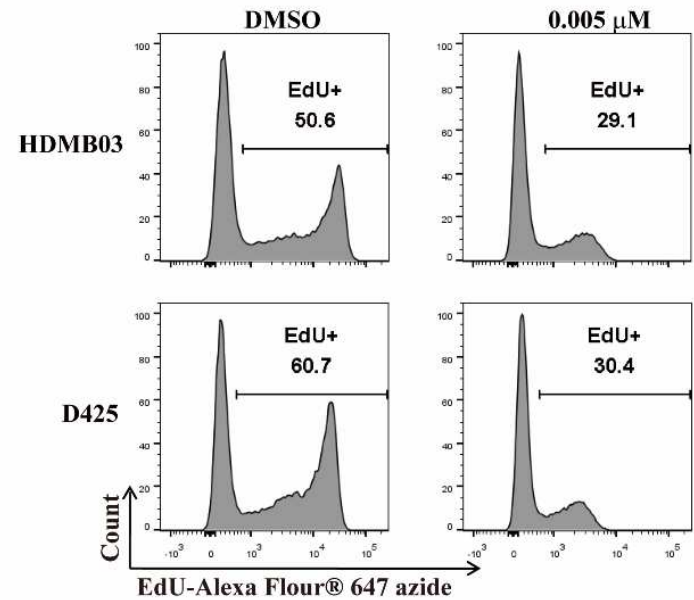
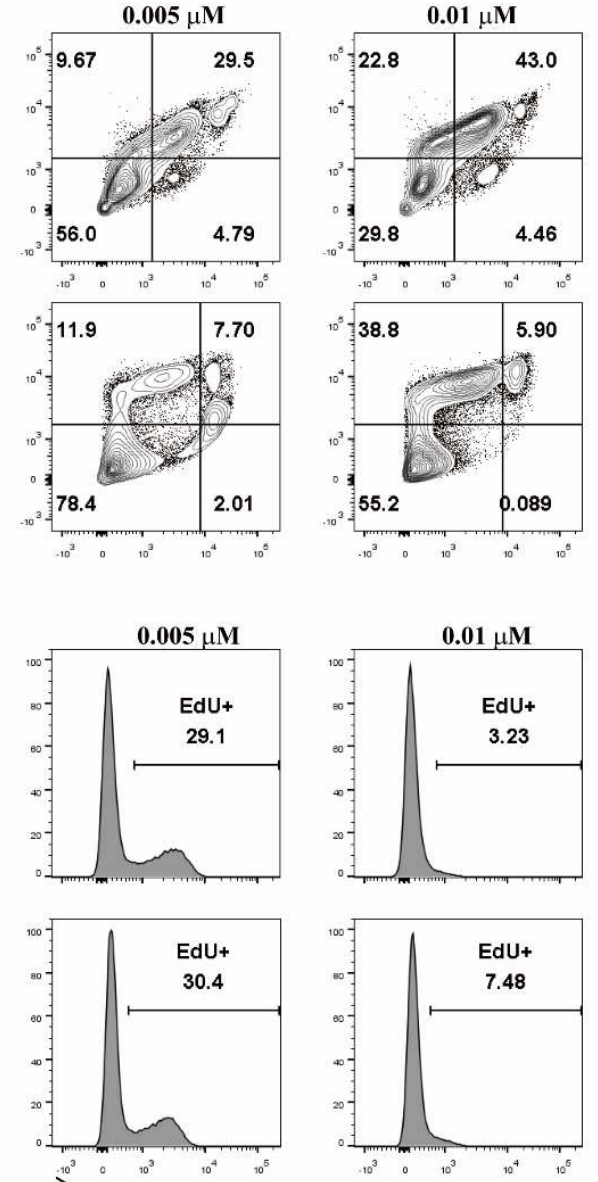
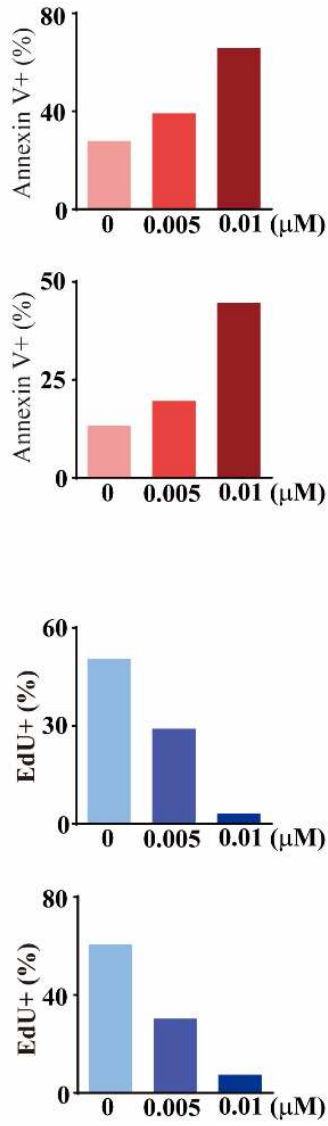
Figure 4
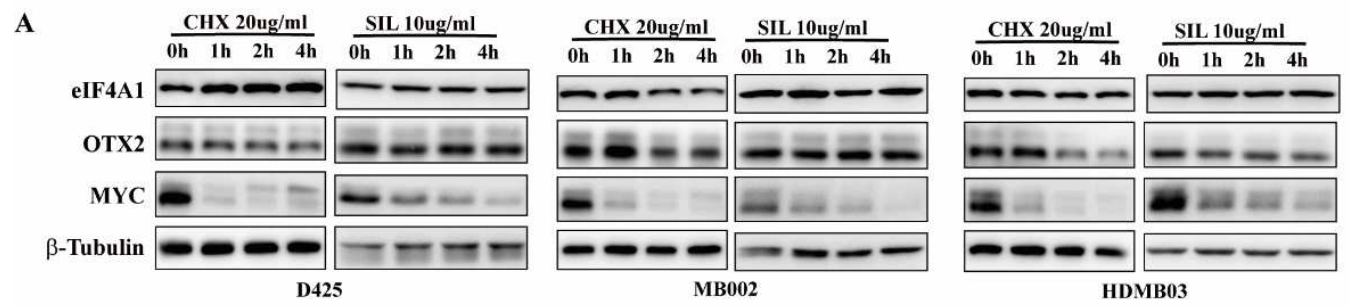

B
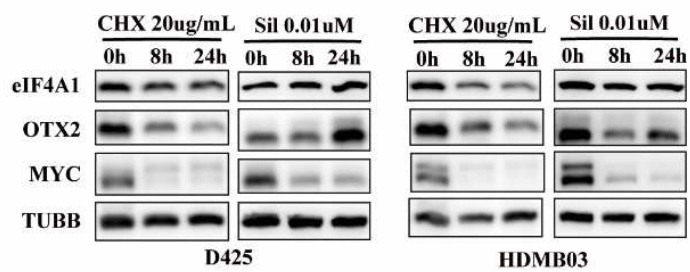

C

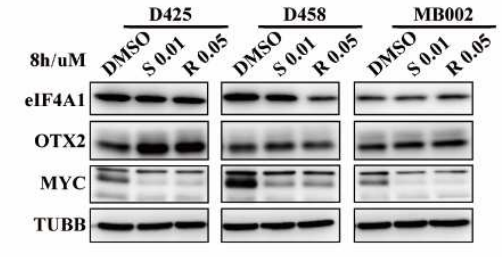

D

MYC

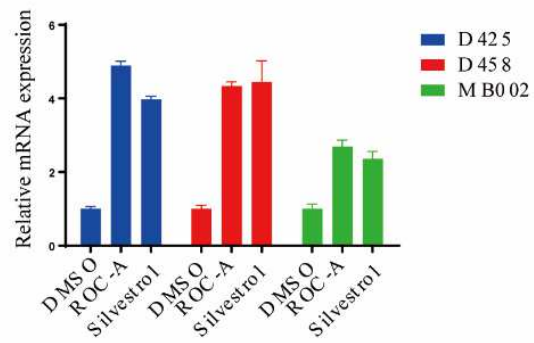

E

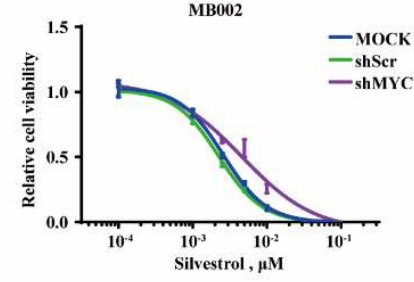

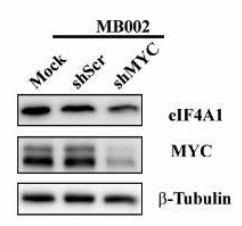

- - -Tubulin
OTX2

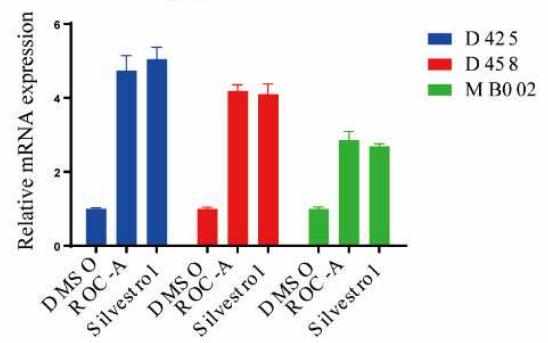

F

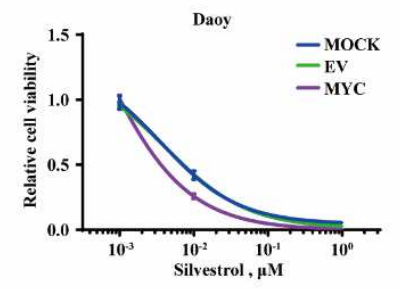

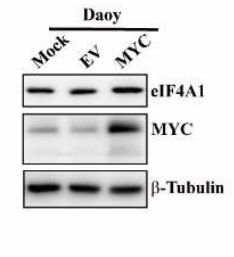


Fig. 5

A

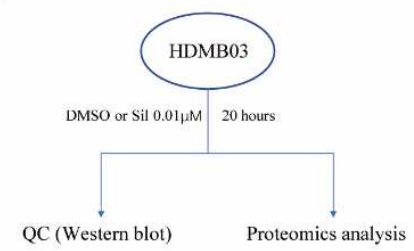

D
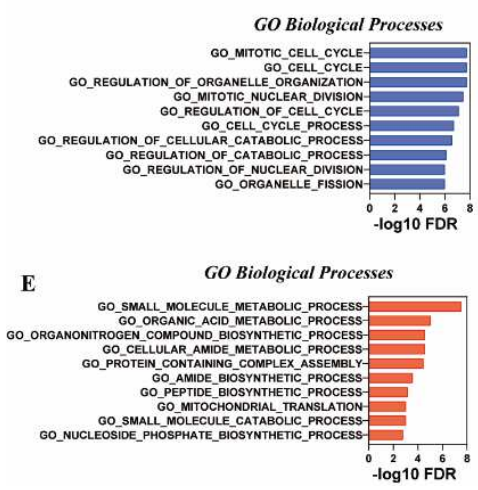

B

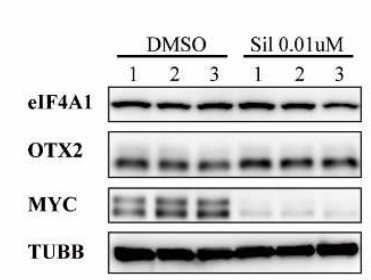

C
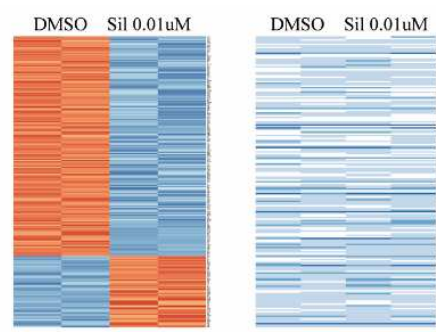

\begin{tabular}{ccccc}
\hline & -0.5 & 0 & 0.5 & 1
\end{tabular}

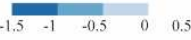

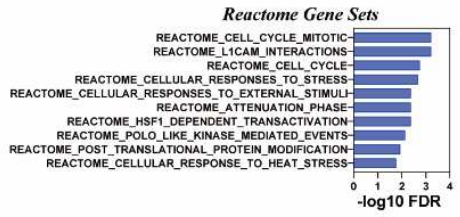

Reactome Gene Sets

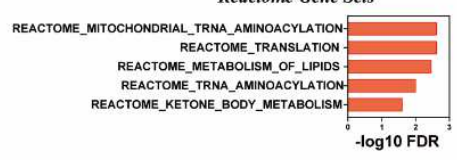

Hallmark Gene Sets

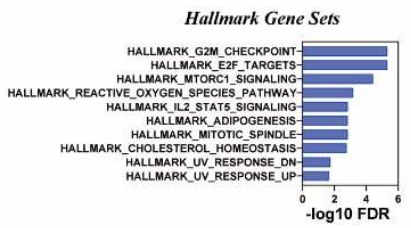

Hallmark Gene Sets HaUMMPXY

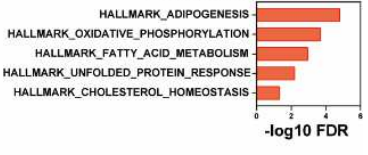


Figures

A

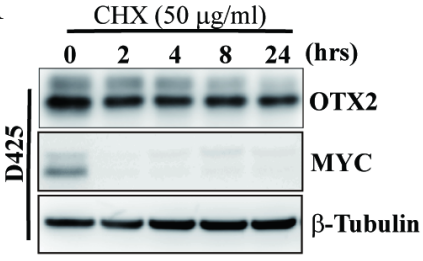

C

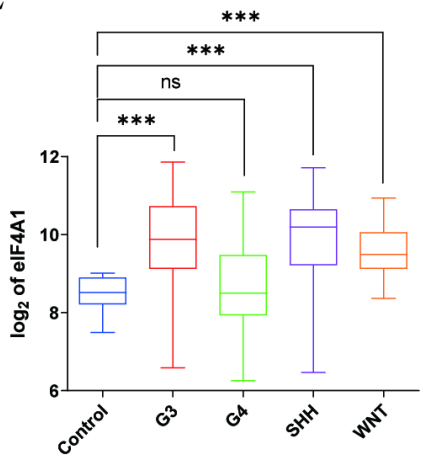

D

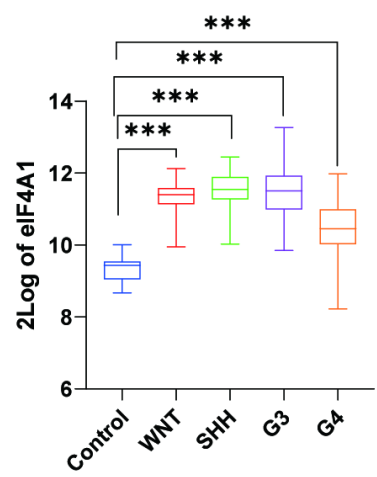

E

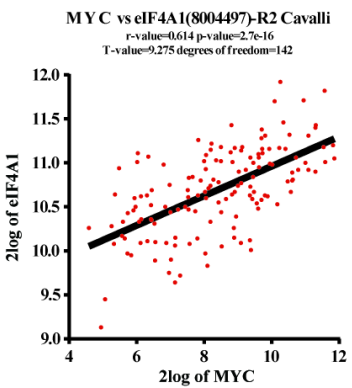

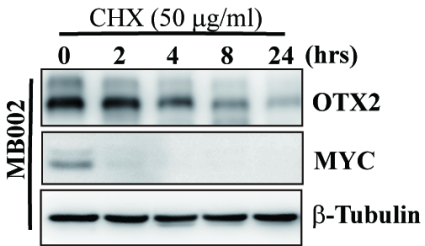

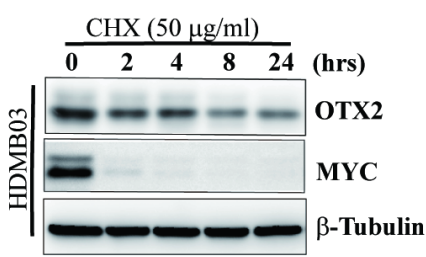

B

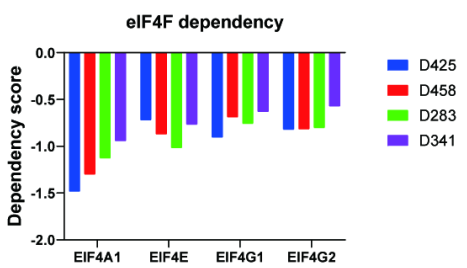

R2 Pomeroy database
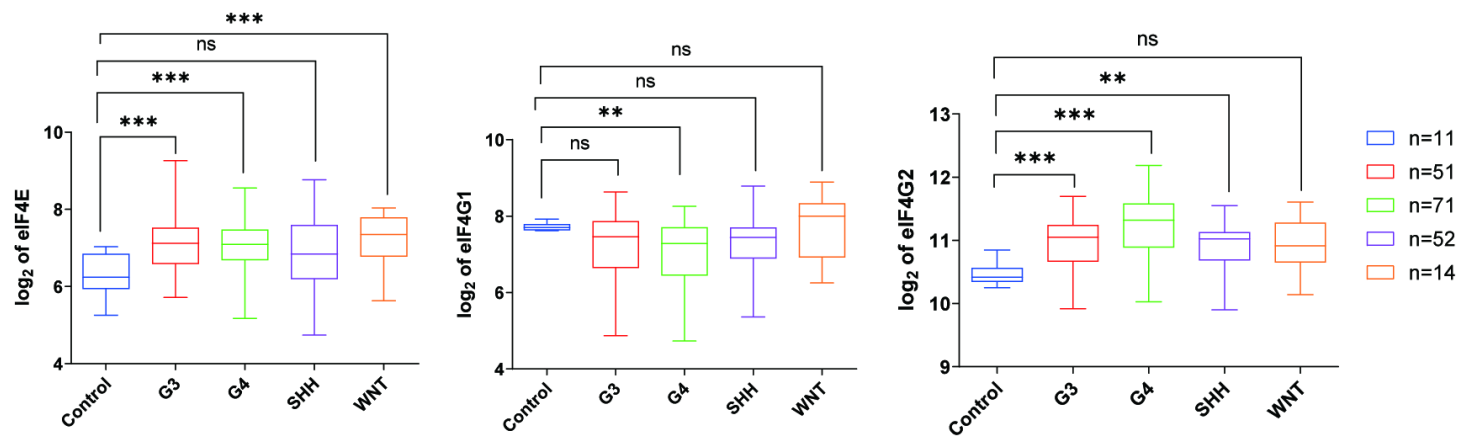

R2 U133P2 database
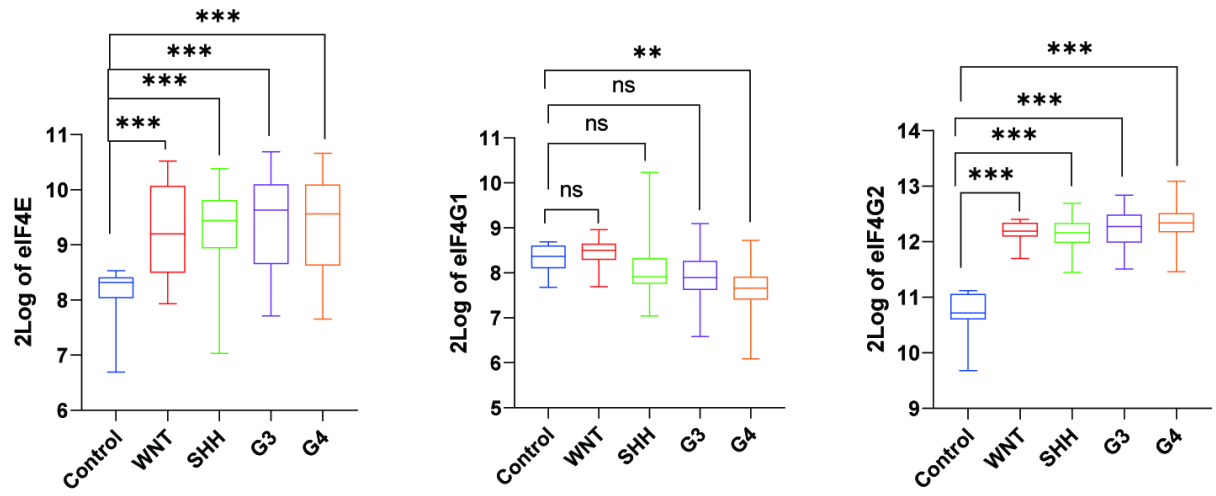

$\square \mathrm{n}=9$

$\square \mathrm{n}=25$

$\square \mathrm{n}=69$

$\square \mathrm{n}=72$

$\square \mathrm{n}=130$

Figure 1
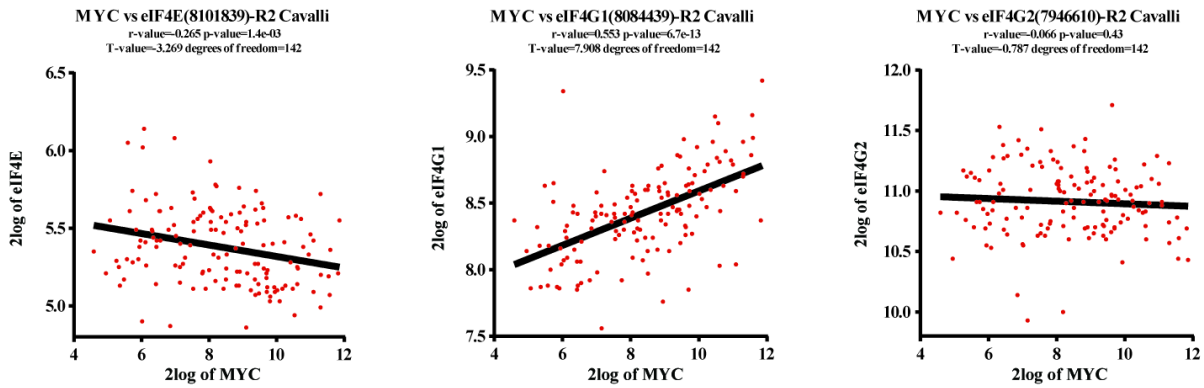

MYC is highly correlated with elF4A1 in Group $3 \mathrm{MB}$. (A) Western blots for the protein levels of MYC and OTX2 in Group 3 MB cell lines HDMB03, MB002 and D425 treated with $50 \mathrm{ng} / \mathrm{mL}$ cycloheximide (CHX). Immunoblot of $\beta$-tubulin is used as a loading control. (B) The dependency score of 5 genes in 4 G3-MB cell lines. (C) The box plots showing the log2 fold changes of mRNA expression levels of elF4F complex members (from left to right: elF4A1, elF4E, elF4G1, elF4G2) compared normal cerebellum with different 
subtype MBs of Pomeroy database. (D) The box plots showing the log2 fold changes of mRNA expression levels of elF4F complex members (from left to right: elF4A1, elF4E, elF4G1, elF4G2) compared normal cerebellum with different subtype MBs of U133p2 database. (E) Scatterplots for the expression correlation of MYC with different subunits of elF4F complex of MB patients in Cavalli database $(n=763)$. Spearman rank correlation test, $\mathrm{Rs}=$ Spearman rank correlation coefficient. All box plots are plotted as mean \pm S.D. *, $p<0.05 ; * \star, p<0.01 ; * \star \star, p<0.001$ by two-tailed Student's $t$ test. MB, medulloblastoma. A

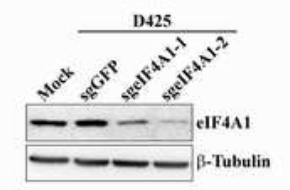

B

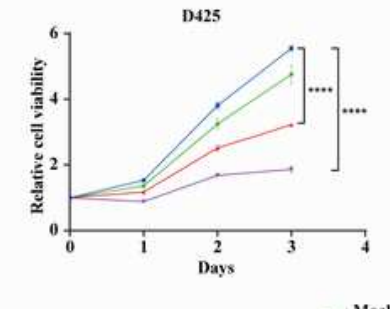

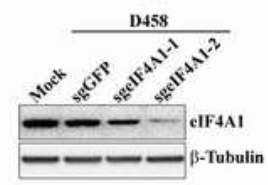

D458

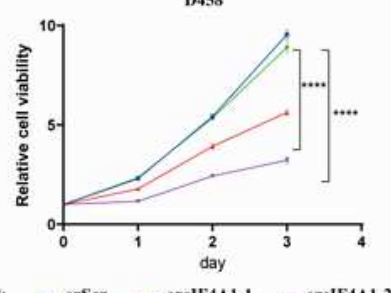

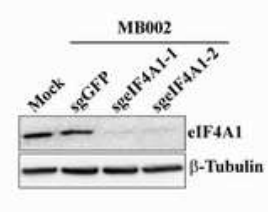

MB002

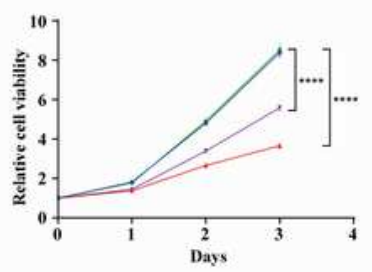

IF $4 \mathrm{A1}-2$
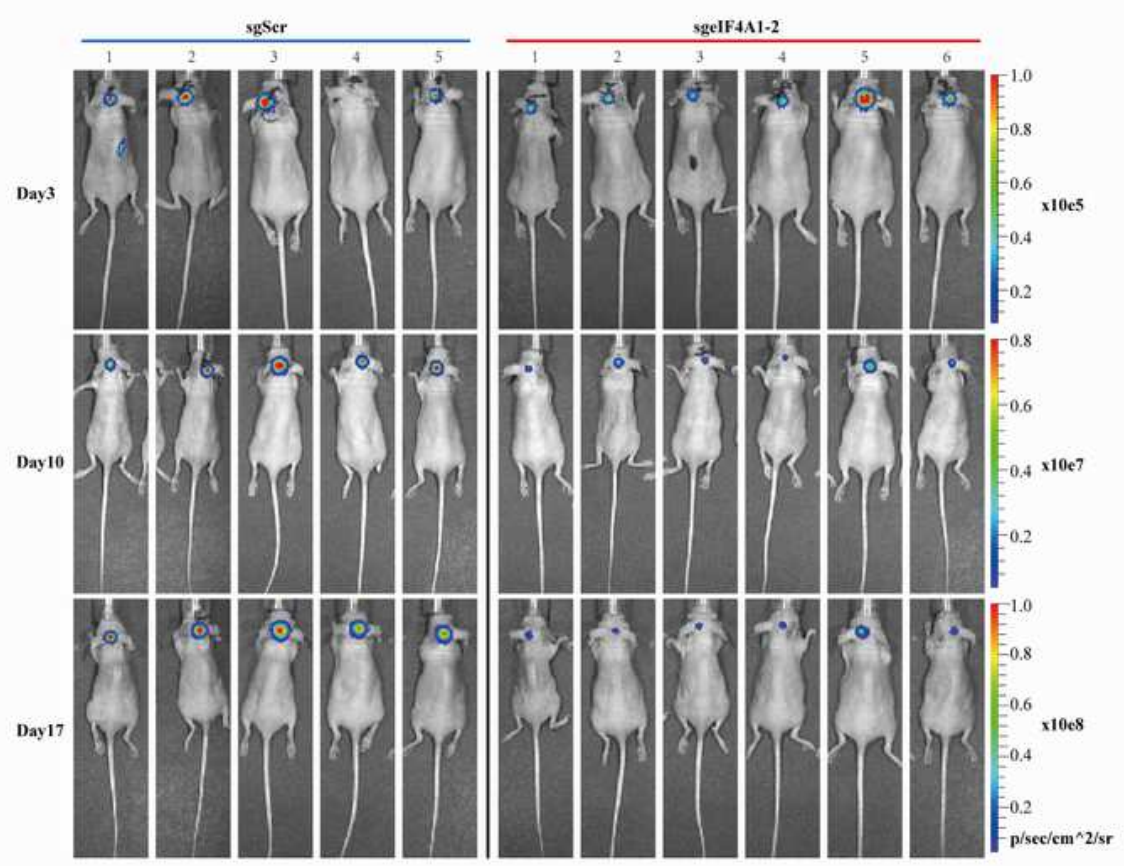

D

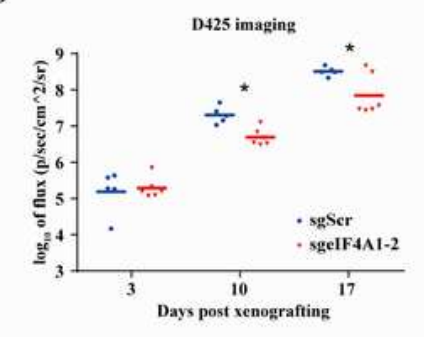

E

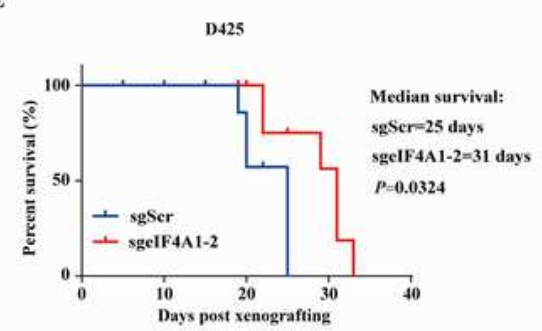


Knock-out elF4A1 can inhibit MB cell proliferation in vitro and xenograft growth in vivo. (A-B) CRISPR/Cas9-mediated deletion of the elF4A1 inhibits D425 cell growth in vitro. (C-D) elF4A1 knock-out (sgelF4A1) or control (sgScr) D425 cells were inoculated orthotopically into the cerebellum of nude mice. $\mathrm{BLI}$ images of photon fluxes of brain tumors at different times were shown (C). The quantification of fluorescence fluxes in B was compared (D). The survival curve of mice bearing sgelF4A1 or sgScr D425 MB tumors were shown (E). Mice implanted with sgelF4A1 D425 tumors display longer survival time with no effect on weight. ( $n=6$ for sgelF4A1 group and $n=5$ for sgScr group). Gehan-Breslow-Wilcoxon test for survival comparison. Two-tailed Student's t test for bioluminescence comparison. sgScr, scrambled sgRNA. 
A

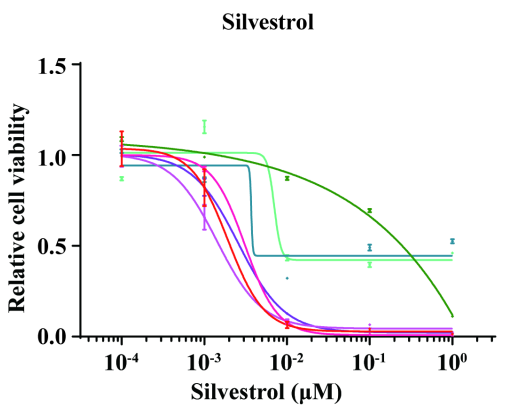

B

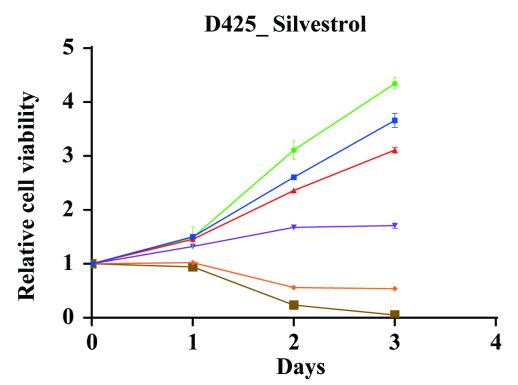

Roc-A
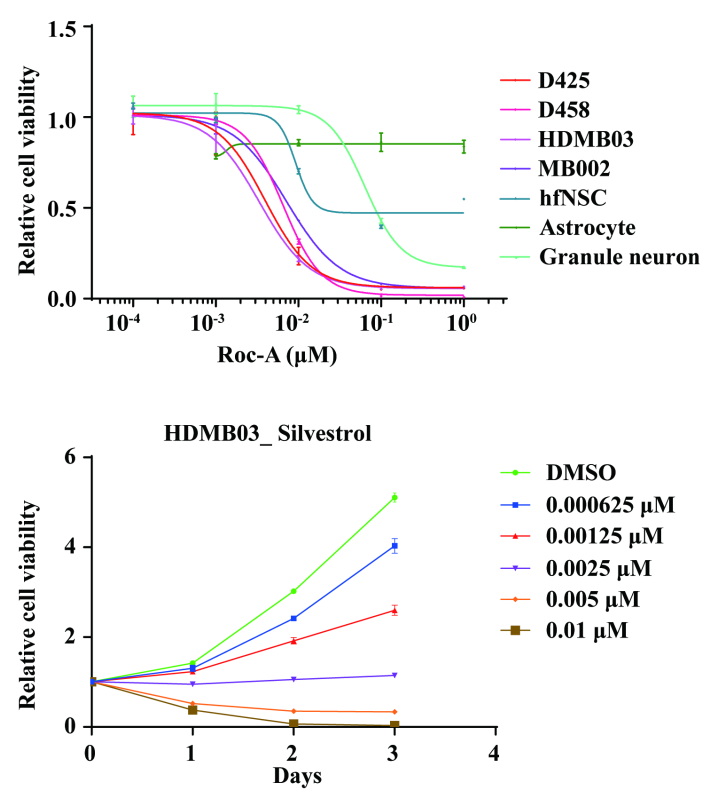

C
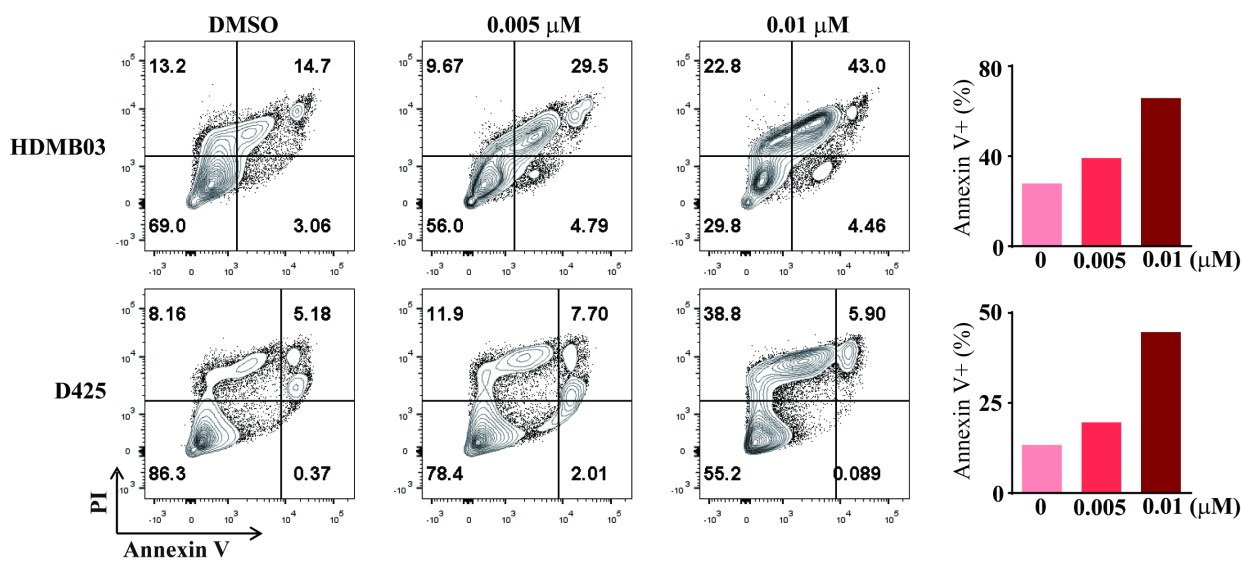

D
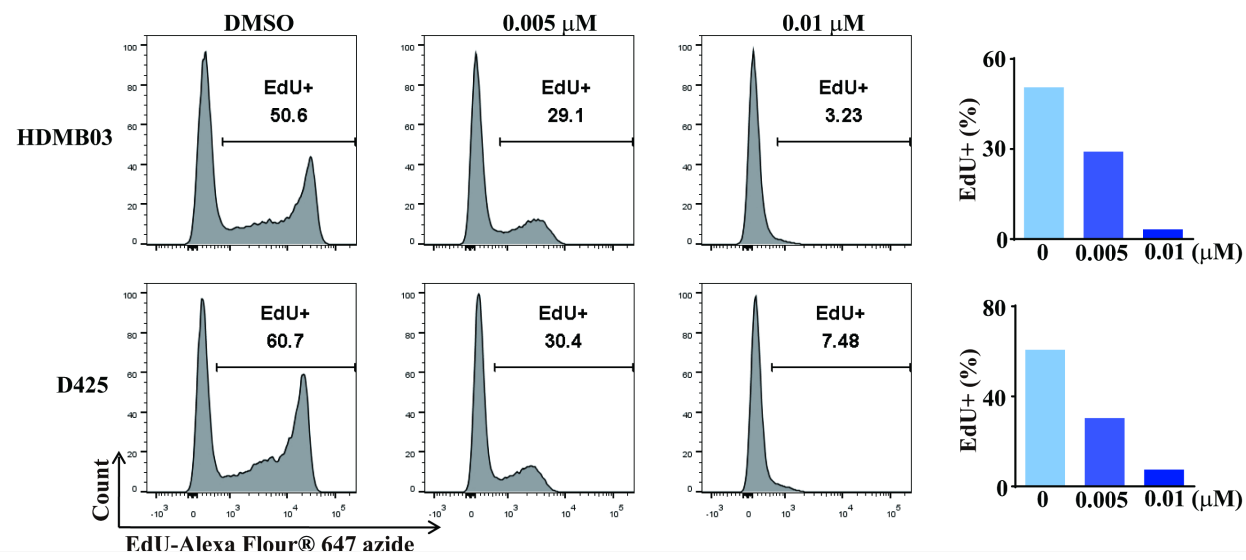

\section{Figure 3}

Validation of the inhibitory effects of elF4A1 inhibitor Silvestrol in MYC-amplified MB. (A) Cell viability was detected in different type of cells after treated with increasing doses of elF4A1 inhibitor (left, Silvestrol; right, Roc-A). (B) Cell viability of D425 (left) or HDMB03 (right) was detected when treated with increasing doses of elF4A1 inhibitor silvestrol for indicated times. (C-D®Apoptosis and proliferation were measured by FACS flow cytometry. Pecentage of Annexin $V$ positive (C) or EdU positive (D) cells of 
Silvestrol treated ( 0.005 or $0.01 \mu \mathrm{M}) \mathrm{HDMB} 03$ and D425 cells were shown. All experiments were repeated at least three times with triplicate samples. Data are plotted as means \pm s.d. ${ }^{*}, p<0.05 ; * \star, p<0.01 ; \star \star \star$, $\mathrm{p}<0.001$ by two-tailed Student's t test.

A

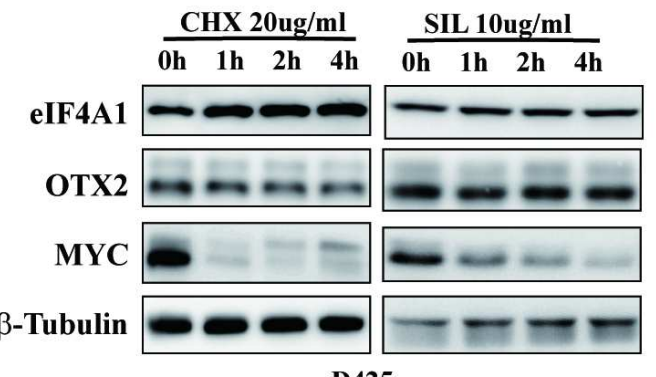

D425

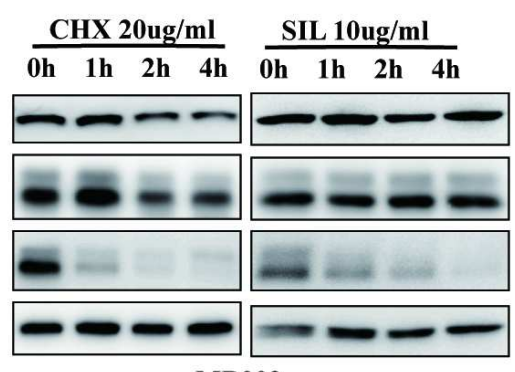

MB002

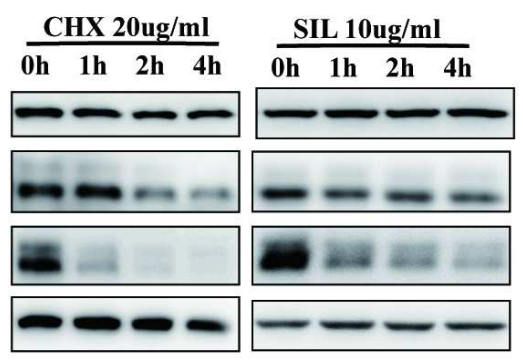

HDMB03

B

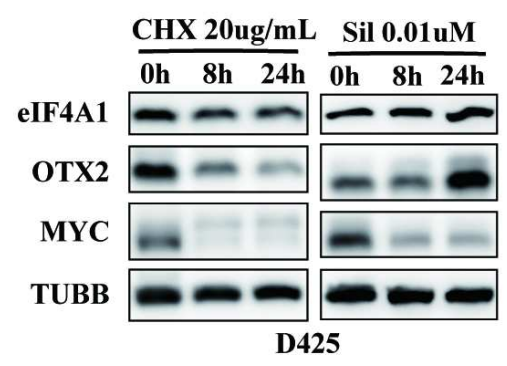

D425

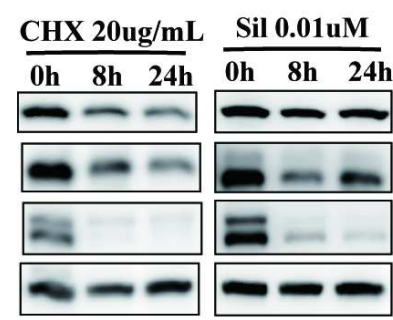

HDMB03

C

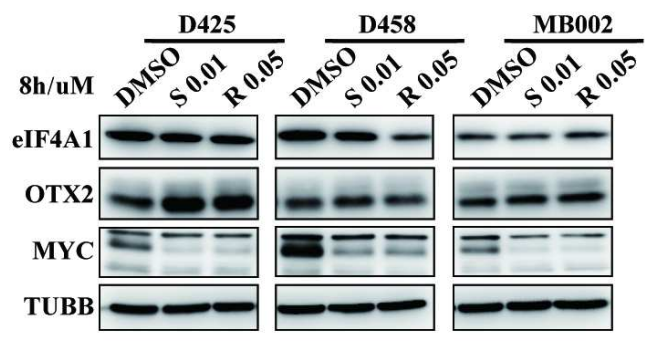

D
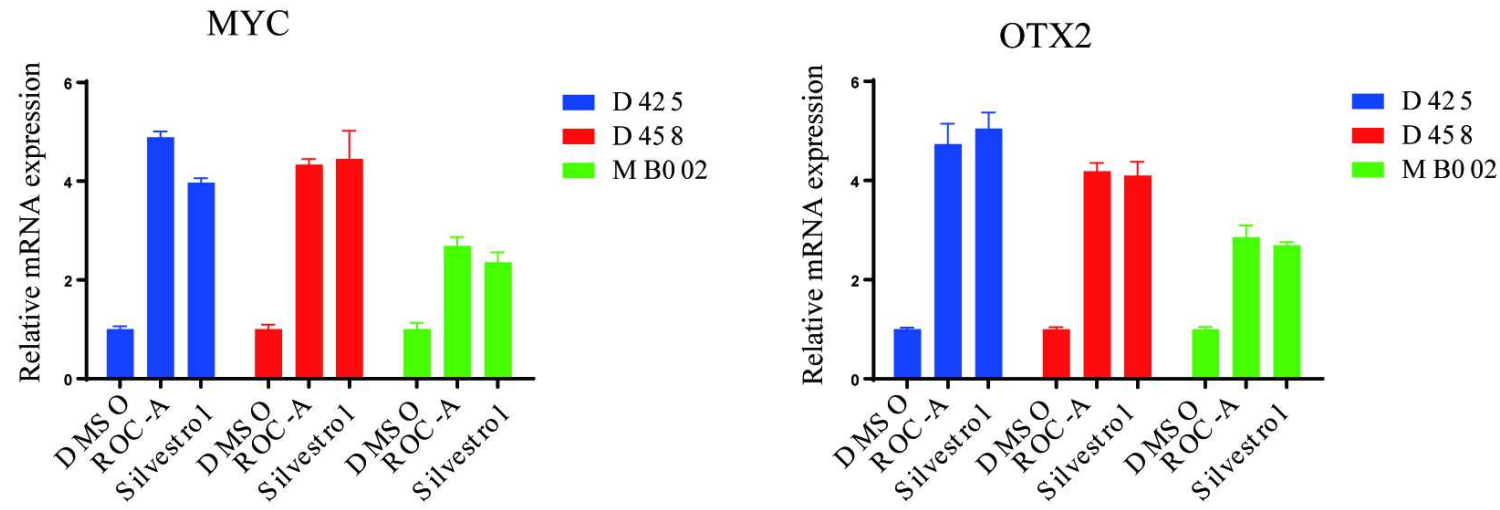

$\mathbf{E}$
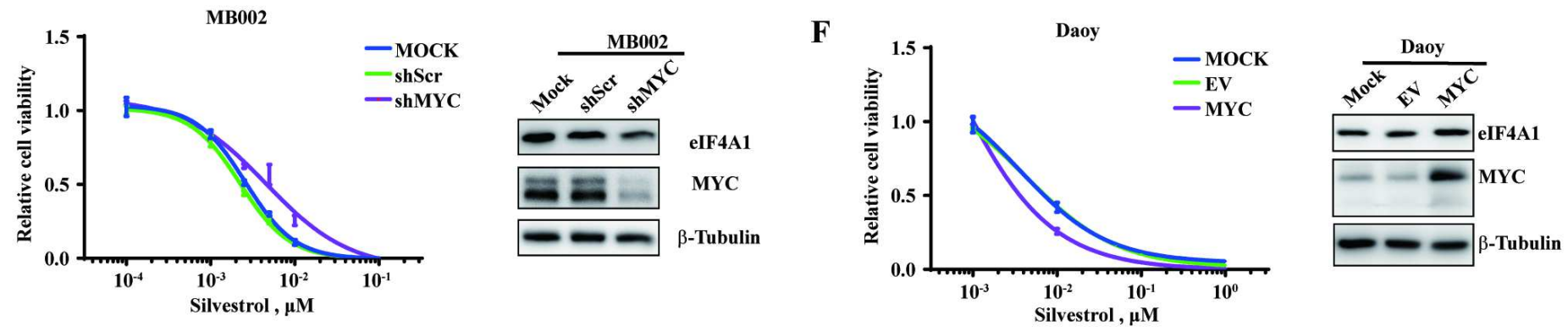

Figure 4

MYC play an essential role in inhibition of MB treated with Silvestrol. (A) Immunoblot was applied to detect MYC $\triangle 0 T X 2$ expression of D425, MB002 and HDMB03 cells treated with Silvestrol $(10 \mu \mathrm{g} / \mathrm{mL})$ or $\mathrm{CHX}(20 \mu \mathrm{g} / \mathrm{mL})$ for 0-4hrs. (B) Immunoblot was applied to detect MYC $₫$ OTX2 expression of D425, 
MB002 and HDMB03 cells treated with Silvestrol $(10 \mu \mathrm{g} / \mathrm{mL})$ or $\mathrm{CHX}(20 \mu \mathrm{g} / \mathrm{mL})$ for 0-24hrs. (C) Immunoblot was applied to detect MYC囚OTX2 expression of D425, MB002 and HDMB03 cells treated with Silvestrol $(10 \mu \mathrm{g} / \mathrm{mL})$ or ROC-A (50 $\mu \mathrm{g} / \mathrm{mL})$. (D) Q-PCR for mRNA levels of MYC in D425, D458 and MB002 cells treated with ROC-A or silvestrol. (E-F) Cell viability was detected in MB002 cells transfected with shMYC or scrambled shRNA (shScr) (D), or in Daoy cells overexpressing MYC or empty vector (EV) (E). Western blots for the protein levels of elF4A1 in MB002 or Daoy cells expressing shRNAs against MYC (shMYC) or scrambled shRNA (shScr) were present at the left respectively. Beta-Tubulin was used as loading control.

A

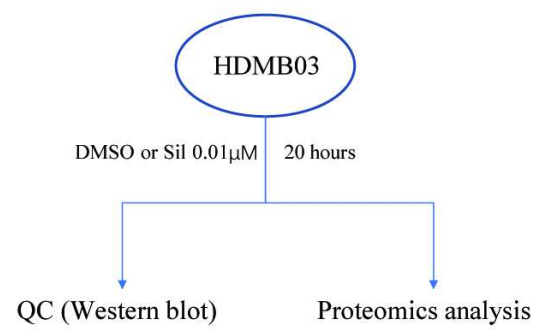

D

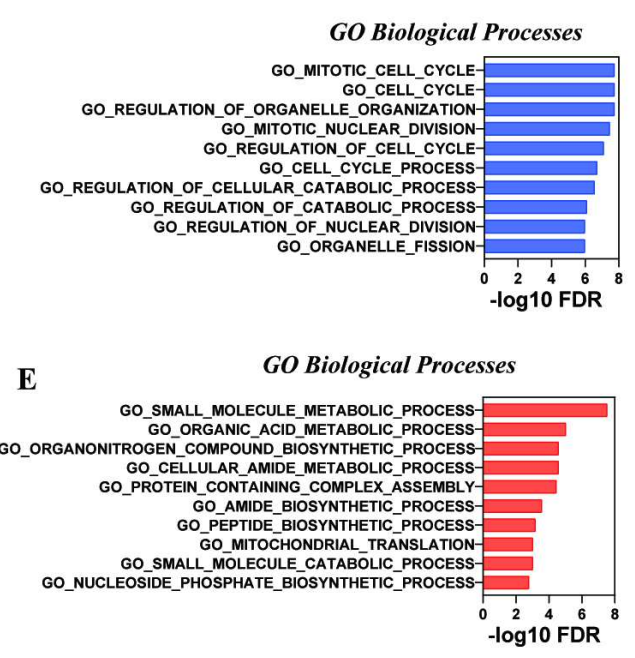

B

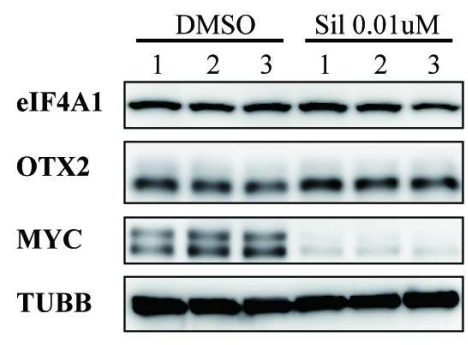

C

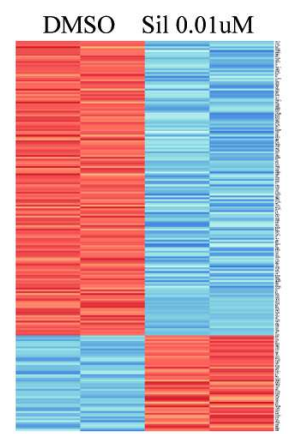

DMSO Sil 0.01uM

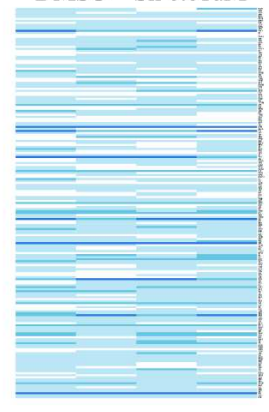

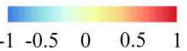

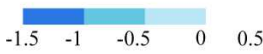

Hallmark Gene Sets
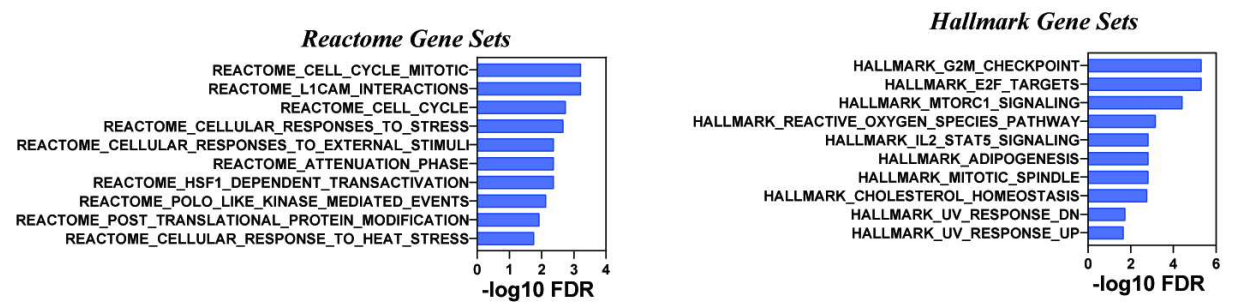

HALLMARK_MTORC1_SIGNALING

LLMARK_IL2_STAT5_SIGNALINGHOLESTEROL_HOMEOSTASIS HALLMARK_UV_RESPONSE_DN-

${ }^{-} \log ^{2} 10$ FDR

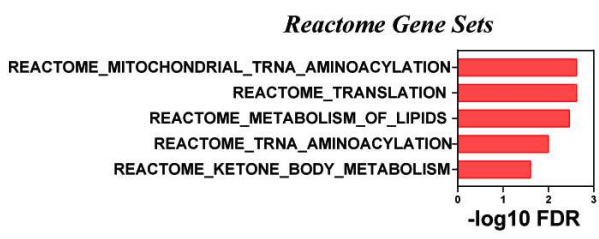

Figure 5

The proteomic expression of MB was modulated by treatment with Silvestrol. (A) HDM03 cells treated with DMSO or Sil were send for QC-WB or proteomic analysis at the same time. (B) Western blot result of QC. (C) Heatmap of proteomic analysis was shown left and the heatmap of gene dependency were shown right. (D) The function analysis of genes down regulated by silvestrol. (E) The function analysis of genes up regulated by silvestrol. 